\title{
On Computing Belief Change Operations using Quantified Boolean Formulas*
}

\author{
James P. Delgrande \\ School of Computing Science \\ Simon Fraser University \\ Burnaby, B.C. \\ Canada V5A 1S6 \\ jim@cs.sfu.ca
}

\author{
Torsten Schaub ${ }^{\dagger}$ \\ Institut für Informatik \\ Universität Potsdam \\ Postfach 900327 \\ D-14439 Potsdam, Germany \\ torsten@cs.uni-potsdam.de
}

\author{
Hans Tompits, Stefan Woltran \\ Institut für Informationssysteme 184/3 \\ Technische Universität Wien \\ Favoritenstraße 9-11 \\ A-1040 Vienna, Austria \\ \{tompits,stefan\}@kr.tuwien.ac.at
}

\begin{abstract}
In this paper, we show how an approach to belief revision and belief contraction can be axiomatised by means of quantified Boolean formulas. Specifically, we consider the approach of belief change scenarios, a general framework that has been introduced for expressing different forms of belief change. The essential idea is that for a belief change scenario $(K, R, C)$, the set of formulas $K$, representing the knowledge base, is modified so that the sets of formulas $R$ and $C$ are respectively true in, and consistent with the result. By restricting the form of a belief change scenario, one obtains specific belief change operators including belief revision, contraction, update, and merging. For both the general approach and for specific operators, we give a quantified Boolean formula such that satisfying truth assignments to the free variables correspond to belief change extensions in the original approach. Hence, we reduce the problem of determining the results of a belief change operation to that of satisfiability. This approach has several benefits. First, it furnishes an axiomatic specification of belief change with respect to belief change scenarios. This then leads to further insight into the belief change framework.
\end{abstract}

*A preliminary version of this paper appeared in the Proceedings of the Sixth European Conference on Symbolic and Quantitative Approaches to Reasoning with Uncertainty (ECSQARU 2001). This work was partially supported by the Austrian Science Fund Project under grants Z29-N04 and P15068-INF, as well as a Canadian NSERC Research Grant.

${ }^{\dagger}$ Affi liated with the School of Computing Science at Simon Fraser University, Burnaby, Canada. 
Second, this axiomatisation allows us to identify strict complexity bounds for the considered reasoning tasks. Third, we have implemented these different forms of belief change by means of existing solvers for quantified Boolean formulas. As well, it appears that this approach may be straightforwardly applied to other specific approaches to belief change.

\section{Introduction}

In previous work, Delgrande and Schaub [12] developed a consistency-based framework for expressing belief change operators. The basic idea with respect to belief revision is that, given a knowledge base $K$ and a sentence $\alpha$ for revision, $K$ and $\alpha$ are fi rst expressed in disjoint languages, then the languages are coerced (via a maximisation process) to agree on truth values of atoms wherever consistently possible, and fi nally the result is then expressed back in the original language. Informally, in the maximisation step, models of $K$ are syntactically forced to correlate with those of $\alpha$ insofar as consistently possible. The inherent nondeterminism of the maximisation process gives rise to two notions of revision: In choice revision, one such belief change extension is selected as the revised state; in general (skeptical) revision, the revised state consists of the intersection of all such extensions. Belief contraction is defi ned similarly.

In this paper, we discuss a method to implement this approach to belief change, based on reductions to quantifi ed Boolean formulas. By a quantifi ed Boolean formula (or QBF for short) one understands a formula which is constructed like an ordinary propositional formula, except that quantifi ers ranging over propositional variables may also occur. Quantifi ed Boolean formulas thus belong to the language of second-order logic. As well, they allow a compact representation of a large class of problems. This latter point is reflected by the fact that the evaluation problem of QBFs - i.e., the problem of determining the truth of a given QBF-is PSPACE-complete, whilst the evaluation problem of QBFs having prenex normal form with $i-1$ alternating (groups of) quantifi ers is complete for the $i$-th level of the polynomial hierarchy [50, 59].

The general mechanism of our approach is to translate (in polynomial time) a given reasoning task into the evaluation problem for QBFs and then use a QBF evaluator to compute the resultant instances. The existence of effi cient QBF solvers, such as the systems developed by Cadoli et al. [4], Rintanen [46], Feldmann et al. [19], or Giunchiglia et al. [24], makes such a rapid prototyping approach practicably applicable. A similar approach for solving various reasoning tasks belonging to the area of nonmonotonic reasoning has been realised in the system QUIP $[15,41,17]$. This prototype implementation currently handles the computation of the main reasoning tasks for logic-based abduction, default logic, several types of modal nonmonotonic logics, and equilibrium logic, a generalisation of the stable model semantics for logic programs. We have implemented the translations for belief change problems by incorporating them into the system QUIP.

Reduction methods to QBFs naturally generalise similar approaches for problems in NP; these latter problems can in turn be solved by translating them (in polynomial time) to SAT, the satisfi ability problem of classical propositional logic (an application of this kind in Artifi cial Intelligence is described, e.g., by Kautz and Selman [26]). Besides the implementation of different nonmonotonic reasoning tasks as realised by the system QUIP, successful applications based on reductions to QBFs have also been applied to conditional planning [45].

There are several reasons why we are interested in a reformulation of the belief change approach of Delgrande and Schaub [12] using QBFs. First, it provides a straightforward implemen- 
tation of the general framework by appeal to extant QBF solvers. Second, in the original approach, several steps were expressed at the metalevel. In particular, there is a metatheoretic step in which pairs of atoms are asserted to be equivalent wherever consistent. Here in contrast, we obtain an object-level representation of the approach. In fact, we provide an axiomatisation of the original belief change method in terms of QBFs by constructing suitable translation schemas such that there is a one-to-one correspondence between the satisfying assignments to the free propositional atoms of the QBFs and the belief change extensions obtained in the original framework. This in turn leads to further insight into the original approach. Finally, the expression of belief change problems in terms of QBFs gives a direct way to estimate the computational complexity of the considered reasoning tasks. More specifi cally, by using the respective QBF encodings, we show that reasoning from choice revision is complete for $\Sigma_{2}^{P}$, and, dually, reasoning from skeptical revision is complete for $\Pi_{2}^{P}$. Additionally, we also discuss the complexity of other decision problems associated with belief change. In this regard, we generalise and improve on earlier reported results [11].

In the next section, we briefly introduce notions of belief change as well as those aspects of belief change scenarios that interest us. In Section 3, we similarly introduce quantifi ed Boolean formulas. Section 4 gives the polynomial-time constructible reductions of the relevant reasoning tasks into QBFs. Section 5 discusses complexity issues, while Section 6 briefly sketches our implementation of the reductions. Section 7 supplies some concluding remarks.

\section{Belief Change and Belief Change Scenarios}

\subsection{Basic Notation}

We deal with propositional languages and use the logical symbols $\top, \perp, \neg, \vee, \wedge, \rightarrow$, and $\equiv$ to construct formulas in the standard way. We write $\mathcal{L}_{\mathcal{P}}$ to denote a language over an alphabet $\mathcal{P}$ of propositional variables or atoms. Formulas are denoted by lower-case Greek letters (possibly with subscripts). Disjunctions of form $\bigvee_{i \in I} \psi_{i}$ are assumed to stand for the logical constant $\perp$ whenever $I=\emptyset$, and likewise conjunctions of form $\bigwedge_{i \in I} \psi_{i}$ with $I=\emptyset$ stand for $\top$. A literal, $L$, is either an atom $p$ (a positive literal) or a negated atom $\neg p$ (a negative literal). The set of all atoms occurring in a formula $\phi$ is denoted by $\operatorname{Var}(\phi)$. Similarly, for a set $S$ of formulas, $\operatorname{Var}(S)$ is the set of all atoms occurring in elements of $S$, i.e., $\operatorname{Var}(S)=\bigcup_{\phi \in S} \operatorname{Var}(\phi)$.

The (propositional) derivability operator, $\vdash$, is defi ned in the usual way, and likewise its semantic counterpart, $\models$. The deductive closure of a set $S \subseteq \mathcal{L}_{\mathcal{P}}$ of formulas is given by $C n_{\mathcal{P}}(S)=\left\{\phi \in \mathcal{L}_{\mathcal{P}} \mid S \vdash \phi\right\}$. We say that $S$ is deductively closed iff $S=C n_{\mathcal{P}}(S)$. Furthermore, $S$ is consistent providing $\perp \notin C n_{\mathcal{P}}(S)$. If the language is clear from the context, we usually drop the index " $\mathcal{P}$ " from $C n_{\mathcal{P}}(\cdot)$ and simply write $C n(\cdot)$. Knowledge bases, or, equivalently, belief sets, are initially identifi ed with deductively-closed sets of formulas; later we relax this restriction. We use $K, K_{1}, \ldots$ to denote knowledge bases.

Given an alphabet $\mathcal{P}$, we defi ne a disjoint alphabet $\mathcal{P}$ as $\mathcal{P}^{\prime}=\left\{p^{\prime} \mid p \in \mathcal{P}\right\}$. Then, for $\alpha \in \mathcal{L}_{\mathcal{P}}$, we defi ne $\alpha^{\prime}$ as the result of replacing in $\alpha$ each atom $p$ from $\mathcal{P}$ by the corresponding atom $p^{\prime}$ in $\mathcal{P}^{\prime}$ (so implicitly there is an isomorphism between $\mathcal{P}$ and $\mathcal{P}^{\prime}$ ). This is defi ned analogously for sets of formulas. 


\subsection{General Approaches to Belief Change}

A common approach in belief revision and other belief change functions is to provide a set of rationality postulates that constrain the results of any such function. The AGM approach of Alchourrón, Gärdenfors, and Makinson [1,21] provides the best-known set of such postulates. Belief states are modelled by deductively-closed sets of sentences, called belief sets, where the underlying logic includes classical propositional logic. $K+\alpha$, the expansion of $K$ by $\alpha$, is defi ned to be $C n(K \cup\{\alpha\}) . K_{\perp}$ is the inconsistent belief set (i.e., $K_{\perp}=\mathcal{L}_{\mathcal{P}}$ ).

A revision function, $\dot{+}$, is a mapping from $2^{\mathcal{L}_{\mathcal{P}}} \times \mathcal{L}_{\mathcal{P}}$ to $2^{\mathcal{L}_{\mathcal{P}}}$ satisfying the following postulates:

$(K \dot{+} 1) K \dot{+} \alpha$ is a belief set.

$(K \dot{+} 2) \alpha \in K \dot{+} \alpha$.

$(K \dot{+} 3) K \dot{+} \alpha \subseteq K+\alpha$.

$(K \dot{+} 4)$ If $\neg \alpha \notin K$, then $K+\alpha \subseteq K \dot{+} \alpha$.

$(K \dot{+} 5) K \dot{+} \alpha=K_{\perp}$ iff $\models \neg \alpha$.

$(K \dot{+} 6)$ If $\models \alpha \equiv \beta$, then $K \dot{+} \alpha=K \dot{+} \beta$.

$(K \dot{+} 7) K \dot{+}(\alpha \wedge \beta) \subseteq(K \dot{+} \alpha)+\beta$.

$(K \dot{+} 8)$ If $\neg \beta \notin K \dot{+} \alpha$, then $(K \dot{+} \alpha)+\beta \subseteq K \dot{+}(\alpha \wedge \beta)$.

Informally, these postulates state that the result of revising $K$ by $\alpha$ is a belief set in which $\alpha$ is believed; whenever the result is consistent, revision consists of the expansion of $K$ by $\alpha$; the only time that $K_{\perp}$ is obtained is when $\alpha$ is inconsistent; and revision is independent of the syntactic form of $K$ and $\alpha$. The last two postulates assert that in revising by a conjunction, an expansion with a conjunct is employed where consistent.

Contraction is the dual notion of revision, in which beliefs are retracted but no new beliefs are added. Postulates $(K \dot{-} 1)-(K \dot{-} 8)$ governing a contraction function, denoted $\dot{-}$, are similarly given. The intuition underlying revision and contraction is that an agent receives new information concerning a static world or domain. Katsuno and Mendelzon [25] explore the distinct notions of belief update and erasure in which an agent changes its beliefs in response to changes in its external environment. As well, belief set merging, in which the contents of two belief sets are combined, is addressed for example by Liberatore and Schaerf [33] and Konieczny and Pino Pérez [28].

There has also been work on specifi c revision operators based on the distance between models of a knowledge base and a sentence to be incorporated in the knowledge base [3, 53, 7, 48, 56, 20]. For example, in the approach of Dalal [7], the revision operator measures the distance between interpretations by the number of propositional variables on which the interpretations differ. It is shown that this operator satisfi es the AGM postulates.

Another direction in belief revision is to assume that revision is not carried out on a belief set itself, but rather on a fi nite subset of the theory. Belief change operations would take place with respect to this belief base, while the underlying belief set would correspond to the deductive closure of this base. The notion of belief base revision is proposed by Makinson [36], and independently, with respect to database systems, by Fagin, Ullman, and Vardi [18]. These approaches are fully 
explored by Nebel [39]. While conceptually simple, revision in these approaches frequently relies on arbitrary syntactic distinctions.

There has been some work with respect to implementations. For example, the aforecited distance-based approaches admit straightforward implementations, see [9, 5]. Otherwise, Williams [55] provides an example of a computational model for belief base revision; see also $[52,2]$ for other approaches. The belief change approach discussed in the present paper has also been implemented as a JAVA program [10].

Finally, Liberatore and Schaerf $[31,32]$ discuss a method which is related to our results. Similar to the ideas of Winslett [57], they employ propositional circumscription [37, 34] in order to express several belief-revision operators, specifi cally those defi ned by Borgida [3], Ginsberg [23], Dalal [7], Satoh [48], and Winslett [58]. The primary point of distinction between the present approach and the aforecited works is that we begin with a general framework in which a suite of diverse operators is defi ned (see following), whereas previous work has for the most part only addressed belief revision.

\subsection{Belief Change via Belief Change Scenarios}

In previous work [12], a consistency-based framework for expressing a suite of belief change operators is developed. The intent was to specify an approach that has good formal properties, but that particularly lent itself to implementation. The approach is discussed formally in the next section; here we give an informal introduction to the approach to revision. As a starting point, it is clear that the syntactic form of a sentence does not give a fi rm indication as to which sentences should be included in a revision $K \dot{+} \alpha$. Alternately, one can consider interpretations, and look at the models of $K$ and $\alpha$. Informally, if $K \cup\{\alpha\}$ is unsatisfi able, a model of $K \dot{+} \alpha$ should contain models of $\alpha$, but in a sense retaining aspects of models of $K$ that do not conflict with those of $\alpha$.

We accomplish this by fi rst expressing $K$ and $\alpha$ in different languages, in essence replacing every occurrence of an atomic sentence $p$ in $K$ by a new atomic sentence $p^{\prime}$ yielding knowledge base $K^{\prime}$, and leaving $\alpha$ unchanged. Under this relabelling, the models of $K^{\prime}$ and $\alpha$ are independent and $K^{\prime} \cup\{\alpha\}$ is satisfi able (assuming that both $K$ and $\alpha$ are satisfi able). The models of $K$ and $\alpha$ are linked by asserting that the languages are (with respect to truth conditions) the same wherever consistently possible. That is, for every $p \in \mathcal{P}$, we assert that $p \equiv p^{\prime}$ wherever consistently possible. We obtain a set of such equivalences, call it $E Q$, such that $K^{\prime} \cup\{\alpha\} \cup E Q$ is consistent. A model of $K^{\prime} \cup\{\alpha\} \cup E Q$ then will be a model of $\alpha$ where the truth values of atomic sentences in $K^{\prime}$ and $\alpha$ are linked wherever possible. A candidate "choice" revision of $K$ by $\alpha$ then consists of $K^{\prime} \cup\{\alpha\} \cup E Q$ re-expressed in the original language. General revision corresponds to the intersection of all candidate choice revisions.

For example, consider $K$ and $\alpha$, where

$$
K=C n(\{(p \vee q) \wedge r\}) \quad \text { and } \quad \alpha=(\neg p \vee \neg q) \wedge \neg r \text {. }
$$

Renaming the atoms in $K$ gives $K^{\prime}=C n\left(\left\{\left(p^{\prime} \vee q^{\prime}\right) \wedge r^{\prime}\right\}\right)$. Clearly, $K^{\prime} \cup\{\alpha\}$ is consistent, even though $K \cup\{\alpha\}$ is not. In the step to link the interpretations of $K^{\prime}$ and $\alpha$, we obtain that $C n\left(K^{\prime} \cup\{\alpha\} \cup\left\{p^{\prime} \equiv p, q^{\prime} \equiv q\right\}\right)$ is consistent, but $C n\left(K^{\prime} \cup\{\alpha\} \cup\left\{p^{\prime} \equiv p, q^{\prime} \equiv q, r^{\prime} \equiv r\right\}\right)$ is not. Hence, we take $E Q=\left\{p^{\prime} \equiv p, q^{\prime} \equiv q\right\}$. Intersecting $C n\left(K^{\prime} \cup\{\alpha\} \cup E Q\right)$ with the original language yields $C n(\{(p \equiv \neg q) \wedge \neg r\})$ as the revised knowledge base. 
The general framework allows the expression of contraction and integrity constraints, as well as update, erasure, and merging operations. Signifi cantly, the approach is independent of how the knowledge base and formula for revision are represented. In particular, the original and revised knowledge base can be represented by a formula whose deductive closure gives the corresponding belief set. As well, the scope of a revision (for example) can be restricted to just those propositions common to the knowledge base and sentence for revision. The approach (essentially) satisfi es the AGM postulates [1], with the exception of the revision postulate $(K \dot{+} 8)$ and the contraction postulate $(K \dot{-} 8)$, and the contraction "recovery" postulate $(K \dot{-} 5)$. The approach to belief change is founded on the same intuitions as consistency-based reasoning methodologies in Artifi cial Intelligence. Examples of such systems include Theorist [43], diagnosis from fi rst principles [44], and the assumption-based approach to truth maintenance [8].

\subsection{Formal Elements of the Belief Change Framework}

Following Delgrande and Schaub [12], we defi ne a belief change scenario in language $\mathcal{L}_{\mathcal{P}}$ as a triple $B=(K, R, C)$, where $K, R$, and $C$ are sets of formulas in $\mathcal{L}_{\mathcal{P}}$. Informally, $K$ is a knowledge base that will be changed such that the set $R$ will be implied by the result, and the set $C$ will be consistent with the result. For a base approach to revision we take $C=\emptyset$, and for a base approach to contraction we take $R=\emptyset$.

We extend our notation $\operatorname{Var}(\cdot)$ to belief change scenarios in the obvious way, i.e., for $B=$ $(K, R, C)$, we defi ne $\operatorname{Var}(B)=\operatorname{Var}(K \cup R \cup C)$. In the defi nition below, "maximal" is with respect to set containment (rather than set cardinality). The following defi nition is central:"

Definition 2.1 Let $B=(K, R, C)$ be a belief change scenario in $\mathcal{L}_{\mathcal{P}}$. Define EQ as a maximal set of equivalences $E Q \subseteq\left\{p \equiv p^{\prime} \mid p \in \mathcal{P}\right\}$ such that

$$
K^{\prime} \cup E Q \cup R \cup C \nvdash \perp .
$$

Then,

$$
C n\left(K^{\prime} \cup E Q \cup R\right) \cap \mathcal{L}_{\mathcal{P}}
$$

is $a$ (consistent) belief change extension of $B$.

If there is no such set $E Q$, then $B$ is inconsistent, and $\mathcal{L}_{\mathcal{P}}$ is defined to be the sole (inconsistent) belief change extension of $B$.

So, a (consistent) belief change extension of $B$ is a modification of $K$ in which $R$ is true, and in which $C$ is consistent. We say that $E Q$ determines the belief change extension $C n\left(K^{\prime} \cup E Q \cup R\right) \cap \mathcal{L}_{\mathcal{P}}$ of $B$. Clearly, for a given belief change scenario, there may be more than one belief change extension.

Defi nition 2.1 provides a very general framework for specifying belief change. In what follows, we give specifi c defi nitions for the belief change operations revision and contraction. In these defi nitions, we make use of the notion of a selection function, $c$, that for any set $I \neq \emptyset$ has as

\footnotetext{
${ }^{1}$ For simplicity, we adopt a slightly simpler formulation of $C$ here than originally given [12]. Here, we require that members of $C$ are put together in determining a belief change extension; in the original formulation [12], members of $C$ are taken individually in determining a belief change extension-that is, $K^{\prime} \cup E Q \cup R \cup\{\neg \phi\} \forall \perp$ for every $\phi \in C \cup\{\perp\}$. We discuss in Section 4.1 how this straightforward yet more involved extension can be accomplished.
} 
Table 1: (Skeptical) revision examples.

\begin{tabular}{c|c|c|c}
$K^{\prime}$ & $\alpha$ & $E Q$ & $K \dot{+} \alpha$ \\
\hline$p^{\prime} \wedge q^{\prime}$ & $\neg q$ & $\left\{p \equiv p^{\prime}\right\}$ & $p \wedge \neg q$ \\
$\neg p^{\prime} \equiv q^{\prime}$ & $\neg q$ & $\left\{p \equiv p^{\prime}, q \equiv q^{\prime}\right\}$ & $p \wedge \neg q$ \\
$p^{\prime} \vee q^{\prime}$ & $\neg p \vee \neg q$ & $\left\{p \equiv p^{\prime}, q \equiv q^{\prime}\right\}$ & $p \equiv \neg q$ \\
$p^{\prime} \wedge q^{\prime}$ & $\neg p \vee \neg q$ & $\left\{p \equiv p^{\prime}\right\},\left\{q \equiv q^{\prime}\right\}$ & $p \equiv \neg q$
\end{tabular}

value $c(I)$ some element of $I$. These primitive functions can be regarded as inducing selection functions $c^{\prime}$ on belief change scenarios, such that $c^{\prime}(B)$ has as value some belief change extension of $B=(K, R, C)$. This is a slight generalisation of selection functions as found in the AGM approach [21].

Definition 2.2 (Revision) Let $K$ be a knowledge base and $\alpha$ a formula, and let $\left(E_{i}\right)_{i \in I}$ be the family of all belief change extensions of $(K,\{\alpha\}, \emptyset)$. Then,

1. $K \dot{+}_{c} \alpha=E_{i}$ is a choice revision of $K$ by $\alpha$ with respect to some selection function $c$ with $c(I)=i$; and

2. $K \dot{+} \alpha=\bigcap_{i \in I} E_{i}$ is the (skeptical) revision of $K$ by $\alpha$.

Table 1 gives examples of (skeptical) revision. The fi rst column gives the original knowledge base, but with atoms already renamed. The second column gives the revision formula, while the third gives the $E Q$ set(s), and the last column gives the results of the revision. For the fi rst and last column, we give a formula whose deductive closure gives the corresponding belief set.

In detail, for the last example, we wish to determine

$$
\{p \wedge q\} \dot{+}(\neg p \vee \neg q) .
$$

We fi nd maximal sets $E Q \subseteq\left\{p \equiv \not h, q \equiv q^{\prime}\right\}$ such that

$$
\left\{p^{\prime} \wedge q^{\prime}\right\} \cup E Q \cup\{\neg p \vee \neg q\} \cup \emptyset \forall \perp \text {. }
$$

We get two such sets of equivalences, viz. $E Q_{1}=\left\{p \equiv p^{\prime}\right\}$ and $E Q_{2}=\left\{q \equiv q^{\prime}\right\}$. Accordingly, we obtain

$$
\{p \wedge q\} \dot{+}(\neg p \vee \neg q)=\bigcap_{i=1,2} C n\left(\left\{p^{\prime} \wedge q^{\prime}\right\} \cup E Q_{i} \cup\{\neg p \vee \neg q\}\right) \cap \mathcal{L}_{\mathcal{P}}
$$

In addition to $(\neg p \vee \neg q)$, we get $(p \vee q)$, jointly implying $(p \equiv \neg q)$.

Contraction is defi ned similarly to revision.

Definition 2.3 (Contraction) Let $K$ be a knowledge base and $\alpha$ a formula, and let $\left(E_{i}\right)_{i \in I}$ be the family of all belief change extensions of $(K, \emptyset,\{\neg \alpha\})$. Then,

1. $K \dot{-}_{c} \alpha=E_{i}$ is a choice contraction of $K$ by $\alpha$ with respect to some selection function $c$ with $c(I)=i$; and 
Table 2: (Skeptical) contraction examples.

\begin{tabular}{c|c|c|c}
$K^{\prime}$ & $\alpha$ & $E Q$ & $K \dot{-} \alpha$ \\
\hline$p^{\prime} \wedge q^{\prime}$ & $q$ & $\left\{p \equiv p^{\prime}\right\}$ & $p$ \\
$p^{\prime} \wedge q^{\prime} \wedge r^{\prime}$ & $p \vee q$ & $\left\{r \equiv r^{\prime}\right\}$ & $r$ \\
$p^{\prime} \vee q^{\prime}$ & $p \wedge q$ & $\left\{p \equiv p^{\prime}, q \equiv q^{\prime}\right\}$ & $p \vee q$ \\
$p^{\prime} \wedge q^{\prime}$ & $p \wedge q$ & $\left\{p \equiv p^{\prime}\right\},\left\{q \equiv q^{\prime}\right\}$ & $p \vee q$
\end{tabular}

2. $K \dot{-} \alpha=\bigcap_{i \in I} E_{i}$ is the (skeptical) contraction of $K$ by $\alpha$.

We note that the previous revision and contraction operations only partially satisfy Harper's Identity, given by $K \dot{-} \alpha=K \cap(K \dot{+} \neg \alpha)$, viz. they satisfy the relation $K \dot{-} \alpha \subseteq K \cap(K \dot{+} \neg \alpha)$. Hence, in the current approach, revision and contraction are distinct operators, and not, as is usually the case, interdefi nable; see [12] for a fuller discussion.

Table 2 gives examples of (skeptical) contraction, using the same format and conventions as Table 1. In detail, for the fi rst example we wish to determine

$$
\{p \wedge q\} \dot{-} q
$$

We compute the belief change extensions of $(\{p \wedge q\}, \emptyset,\{\neg q\})$. We rename the propositions in $\{p \wedge q\}$ and look for maximal subsets $E Q$ of $\left\{p \equiv p^{\prime}, q \equiv q^{\prime}\right\}$ such that

$$
\left\{p^{\prime} \wedge q^{\prime}\right\} \cup E Q \cup \emptyset \cup\{\neg q\} \forall \perp \text {. }
$$

We obtain $E Q=\left\{p \equiv p^{\prime}\right\}$, yielding

$$
\begin{aligned}
\{p \wedge q\} \dot{-} q & =C n\left(\left\{p^{\prime} \wedge q^{\prime}\right\} \cup\left\{p \equiv p^{\prime}\right\} \cup \emptyset\right) \cap \mathcal{L}_{\mathcal{P}} \\
& =C n(\{p\}) .
\end{aligned}
$$

\section{Quantifi ed Boolean Formulas}

Quantifi ed Boolean formulas (QBFs) generalise ordinary propositional formulas by the admission of quantifi cations over propositional variables. As in fi rst-order logic, quantifi cations are either existential or universal, represented by the existential quantifier $\exists$ and the universal quantifier $\forall$, respectively, and follow the intuitive meaning. For instance, the QBF

$$
\forall p \exists q((p \rightarrow q) \wedge(q \rightarrow p))
$$

evaluates to true, since for all truth assignments to $p$, there exists a truth assignment to $q$, such that the propositional formula $(p \rightarrow q) \wedge(q \rightarrow p)$ evaluates to true. On the other, hand the QBF

$$
\exists p \forall q((p \rightarrow q) \wedge(q \rightarrow p))
$$

evaluates to false. 
In general, QBFs are a conservative extension of classical propositional logic, in the sense that to each QBF we can assign a logically equivalent propositional formula. However, the advantage of QBFs is their compactness: to express a QBF as a logically equivalent propositional formula, one has to face an exponential increase of the formula size, in general. Furthermore, QBFs extend classical propositional logic in such a way that reasoning over truth assignments within the object language can be expressed. A different way to view QBFs is to regard them as a subclass of second-order logic, restricting predicates to be of arity zero, and therefore to consider formulas without function symbols and object variables.

Historically, among the fi rst logical analyses of systems dealing with quantifi ers over propositional variables are the investigations due to Russell ("theory of implication" [47]) and Łukasiewicz and Tarski ("erweiterter Aussagenkalkül" [35]), not to mention the monumental Principia Mathematica [54]. The particular idea of quantifying propositional variables was extended in Leśniewski's system of protothetic logic $[29,49]$ where variables whose values are truth functions are allowed and quantifi cation is defi ned over these variables. In the beginning of the seventies of the last century, propositional quantifi cation came into the spotlight of computer science, in particular in the new and developing fi eld of complexity theory [22], when evaluation problems for QBFs were recognised as the prototypical problems for the polynomial hierarchy [50] as well as for the prominent complexity class PSPACE [38]. Details on the relation between QBFs and complexity theory are given in Section 5.

Formally, the set of quantifi ed Boolean formulas (QBFs) over alphabet $\mathcal{P}$ is inductively defi ned as follows:

1. any propositional variable $p \in \mathcal{P}$ and any logical constant $\perp, \top$ is a QBF;

2. if $\Phi$ is a $\mathrm{QBF}$, then $(\neg \Phi)$ is a $\mathrm{QBF}$;

3. if $\Phi$ and $\Psi$ are QBFs, then $(\Phi \wedge \Psi),(\Phi \vee \Psi),(\Phi \rightarrow \Psi)$, and $(\Phi \equiv \Psi)$ are QBFs;

4. if $p \in \mathcal{P}$ is a propositional variable and $\Phi$ is a QBF, then $(\exists p \Phi)$ and $(\forall p \Phi)$ are QBFs;

5. the only QBFs are those given by $1-4$.

We tacitly assume the usual conventions concerning the omission of parantheses in formulas where no ambiguities can arise. Furthermore, we use upper-case Greek letters as meta-variables for QBFs, whilst lower-case Greek letters stand for propositional formulas (i.e., quantifi er-free QBFs). Our defi nition of quantifi ed Boolean formulas is rather unrestricted in two ways: First, in contrast to the formalisation of QBFs in some papers of the relevant literature, we allow quantifi ers to appear anywhere in a formula. Second, we do not stipulate any restriction on the quantifi cation, i.e., we do not require that a quantifi ed variable $p$ in $\mathrm{Q} p \Phi(\mathrm{Q} \in\{\exists, \forall\})$ occurs in the scope $\Phi$ of $\mathrm{Q} p$. For example, $(\exists p(q \wedge r))$ is a QBF, and so is $(\exists p(\forall p(p \rightarrow q)))$.

The semantics of QBFs is defi ned as follows. First, some ancillary notation. An occurrence of a propositional variable $p$ in a QBF $\Phi$ is free iff it does not appear in the scope of a quantifi cation $\mathrm{Q} p$ $(\mathrm{Q} \in\{\forall, \exists\}$ ), otherwise the occurrence of $p$ is bound. If $\Phi$ contains no free variable occurrences,

\footnotetext{
${ }^{2} \mathrm{~A}$ more elaborate overview on these early historical aspects of propositional quantifi cation can be found in $\S 28$ of Church's Introduction to Mathematical Logic [6].
} 
then $\Phi$ is closed, otherwise $\Phi$ is open. Furthermore, $\Phi\left[p_{1} / \phi_{1}, \ldots, p_{n} / \phi_{n}\right]$ denotes the result of uniformly substituting each free occurrence of a variable $p_{i}$ in $\Phi$ by a formula $\phi_{i}$, for $1 \leq i \leq n$.

By an interpretation, $M$, we understand a set of atoms. Informally, an atom $p$ is true under $M$ iff $p \in M$. In general, the truth value, $\nu_{M}(\Phi)$, of a QBF $\Phi$ under an interpretation $M$ is recursively defi ned as follows:

1. if $\Phi=\top$, then $\nu_{M}(\Phi)=1$, and if $\Phi=\perp$, then $\nu_{M}(\Phi)=0$;

2. if $\Phi=p$ is an atom, then $\nu_{M}(\Phi)=1$ if $p \in M$, and $\nu_{M}(\Phi)=0$ otherwise;

3. if $\Phi=\neg \Psi$, then $\nu_{M}(\Phi)=1-\nu_{M}(\Psi)$;

4. if $\Phi=\left(\Phi_{1} \wedge \Phi_{2}\right)$, then $\nu_{M}(\Phi)=\min \left(\left\{\nu_{M}\left(\Phi_{1}\right), \nu_{M}\left(\Phi_{2}\right)\right\}\right)$;

5. if $\Phi=\left(\Phi_{1} \vee \Phi_{2}\right)$, then $\nu_{M}(\Phi)=\max \left(\left\{\nu_{M}\left(\Phi_{1}\right), \nu_{M}\left(\Phi_{2}\right)\right\}\right)$;

6. if $\Phi=\left(\Phi_{1} \rightarrow \Phi_{2}\right)$, then $\nu_{M}(\Phi)=1$ if $\nu_{M}\left(\Phi_{1}\right) \leq \nu_{M}\left(\Phi_{2}\right)$, and $\nu_{M}(\Phi)=0$ otherwise;

7. if $\Phi=\left(\Phi_{1} \equiv \Phi_{2}\right)$, then $\nu_{M}(\Phi)=1$ if $\nu_{M}\left(\Phi_{1}\right)=\nu_{M}\left(\Phi_{2}\right)$, and $\nu_{M}(\Phi)=0$ otherwise;

8. if $\Phi=\forall p \Psi$, then $\nu_{M}(\Phi)=\nu_{M}(\Psi[p / \top] \wedge \Psi[p / \perp])$; and

9. if $\Phi=\exists p \Psi$, then $\nu_{M}(\Phi)=\nu_{M}(\Psi[p / \top] \vee \Psi[p / \perp])$.

We say that $\Phi$ is true under $M$ iff $\nu_{M}(\Phi)=1$, otherwise $\Phi$ is false under $M$. If $\nu_{M}(\Phi)=1$, then $M$ is a model of $\Phi$. The set of all models of $\Phi$ is denoted by $\operatorname{Mod}(\Phi)$. If $\operatorname{Mod}(\Phi) \neq \emptyset$, then $\Phi$ is said to be satisfiable. If $\Phi$ is true under every interpretation, then $\Phi$ is valid. As usual, we write $=\Phi$ to express that $\Phi$ is valid.

It is easily seen that the truth value of a closed QBF is either true under every interpretation or false under every interpretation, i.e., a closed QBF is either valid or unsatisfi able. In general, the truth value of an arbitrary QBF under an interpretation depends only on its free variables. Hence, without loss of generality, for determining the truth value of QBFs, we may restrict our attention to interpretations which contain only atoms occurring free in the given QBF.

If a closed QBF $\Phi$ is valid, we say that $\Phi$ evaluates to true, and, correspondingly, if $\Phi$ is unsatisfi able, we say that $\Phi$ evaluates to false. Two sets of formulas (i.e., ordinary propositional formulas or QBFs) are logically equivalent iff they possess the same models. Thus, formulas $\Phi$ and $\Psi$ are logically equivalent iff $\Phi \equiv \Psi$ is valid.

In the sequel, we use the following abbreviations in the context of QBFs: For an indexed set $V=\left\{p_{1}, \ldots, p_{n}\right\}$ of propositional variables and a quantifi er $\mathrm{Q} \in\{\forall, \exists\}$, we let $\mathrm{Q} V \Phi$ stand for the formula $\mathrm{Q} p_{1} \mathrm{Q} p_{2} \ldots \mathrm{Q} p_{n} \Phi$. An analogous notation applies if $V$ is a string $p_{1} \ldots p_{n}$ of variables. Moreover, let $S=\left\{\phi_{1}, \ldots, \phi_{n}\right\}$ and $T=\left\{\psi_{1}, \ldots, \psi_{n}\right\}$ be indexed sets of formulas. Then, $S \leq T$ is an abbreviation for $\left\{\phi_{1} \rightarrow \psi_{1}, \ldots, \phi_{n} \rightarrow \psi_{n}\right\}$, and $S \equiv T$ stands for $\left\{\phi_{1} \equiv \psi_{1}, \ldots, \phi_{n} \equiv \psi_{n}\right\}$. Obviously, $S \equiv T$ is logically equivalent to $(S \leq T) \cup(T \leq S)$.

The two set operations $\leq$ and $\equiv$ can of course also be applied in a composed manner. In particular, for $S$ and $T$ as above and $R=\left\{\varphi_{1}, \ldots, \varphi_{n}\right\}$, we will make use of the expression

$$
R \leq(S \equiv T)
$$


abbreviating the set of formulas $\bigcup_{i=1}^{n}\left\{\varphi_{i} \rightarrow\left(\phi_{i} \equiv \psi_{i}\right)\right\}$.

Whenever an indexed set $S$ of formulas is used as a consecutive part of a QBF, we implicitly understand $S$ as the formula $\bigwedge_{\phi \in S} \phi$. In this sense, for $R, S, T$ as in (1), the expression $R \leq(S \equiv$ $T)$ appearing within a QBF is synonymous to the formula $\bigwedge_{i=1}^{n}\left(\varphi_{i} \rightarrow\left(\phi_{i} \equiv \psi_{i}\right)\right)$.

Generally speaking, the operator $\leq$ is a fundamental tool for expressing certain tests on sets of formulas in terms of QBFs. In particular, we use $\leq$ in conjunction with the following task:

Given fi nite sets $S$ and $T$ of formulas, determine all subsets $R \subseteq S$ such that $T \cup R$ is consistent.

This problem can be encoded by a QBF in the following way:

Proposition 3.1 ([51]) Let $S=\left\{\phi_{1}, \ldots, \phi_{n}\right\}$ and $T$ be finite sets of formulas, let $V$ be the set of atoms occurring in $S \cup T$, and let $G=\left\{g_{1}, \ldots, g_{n}\right\}$ be a set of new variables not occurring in $S$ or T. Furthermore, consider any $R \subseteq S$ and any $M \subseteq G$ such that $\phi_{i} \in R$ iff $g_{i} \in M$, for $1 \leq i \leq n$.

Then, $T \cup R$ is consistent iff $M$ is a model of the $Q B F$

$$
\mathcal{C}[T, S]=\exists V(T \wedge(G \leq S)) .
$$

Note that $\mathcal{C}[T, S]$ is an open $\mathrm{QBF}$ having $G$ as its set of free variables. These variables facilitate the selection of those elements of $S$ which determine the sets $R$ such that $T \cup R$ is consistent. Moreover, $\mathcal{C}[T, S]$ is designed to express all potential subsets $R \subseteq S$ such that $T \cup R$ is consistent.

We illustrate the functioning of this encoding on a simple example. Consider $T=\{\neg p \vee \neg q\}$ and $S=\{p, q\}$. For all proper subsets $R$ of $S, T \cup R$ is consistent, but $T \cup S$ is inconsistent. For $S$ as given, we choose

$$
G=\left\{g_{1}, g_{2}\right\}
$$

as corresponding set of guessing variables, which occur free in the encoding $\mathcal{C}[T, S]$, given by

$$
\exists p q\left((\neg p \vee \neg q) \wedge\left(g_{1} \rightarrow p\right) \wedge\left(g_{2} \rightarrow q\right)\right) .
$$

It can be checked that all interpretations $M \subset G$ are models of (2), but the interpretation $M=G$ is not a model of (2). This coincides with the observation that exactly the proper subsets of $S$, viz. $R_{1}=\emptyset, R_{2}=\{p\}$, and $R_{3}=\{q\}$, are consistent with $W$, while $R_{4}=\{p, q\}$ is not.

To express, for example, all maximal such subsets, some additional elements are required. The computation of maximal sets satisfying certain criteria, using QBFs, is discussed in Section 4.

Finally, we note some useful relations concerning the shifting and renaming of quantifi ers, paralleling similar results from standard fi rst-order logic.

Proposition 3.2 Let $p$ and $q$ be atoms, $Q \in\{\forall, \exists\}$, and let $\Phi$ and $\Psi$ be $Q B F s$ such that $\Psi$ does not contain free occurrences of $p$. Then,

$$
\begin{aligned}
& \text { 1. } \models(\neg \exists p \Phi) \equiv \forall p(\neg \Phi) ; \\
& \text { 2. } \models(\neg \forall p \Phi) \equiv \exists p(\neg \Phi) ; \\
& \text { 3. } \models(\Psi \circ \mathrm{Q} p \Phi) \equiv \mathrm{Q} p(\Psi \circ \Phi) \text { for } \circ \in\{\wedge, \vee, \rightarrow\} ; \text { and } \\
& \text { 4. } \models(\mathrm{Q} q \Psi) \equiv(\mathrm{Q} p \Psi[q / p]) .
\end{aligned}
$$

We say that QBF $\Phi$ is in prenex form if $\Phi=\mathrm{Q}_{1} p_{1} \ldots \mathrm{Q}_{n} p_{n} \phi$, where $\mathrm{Q}_{i} \in\{\forall, \exists\}$, for $1 \leq i \leq n$, and $\phi$ is some propositional formula. Proposition 3.2 implies that any QBF $\Psi$ can be effectively transformed into a logical equivalent $\mathrm{QBF} \Psi^{*}$ in prenex form. 


\section{Reductions}

In this section, we present effi cient (polynomial-time constructible) reductions of the relevant reasoning tasks in the context of belief change scenarios into QBFs. More specifi cally, these reductions are constructed in such a way that there is a one-to-one correspondence between belief change extensions and models of the translated QBFs. Based on these reductions, in Section 5, we analyse the computational complexity of the considered reasoning tasks.

Concerning the specific tasks, we deal with the following decision problems and their corresponding search problems:

EXT: Decide whether a given belief change scenario $B$ has some consistent belief change extension.

CHOICE: Given a belief change scenario $B$ and some formula $\phi$, decide whether $\phi$ is contained in at least one consistent belief change extension of $B$.

SKEPTICAL: Given a belief change scenario $B$ and some formula $\phi$, decide whether $\phi$ is contained in all belief change extensions of $B$.

Note that EXT and CHOICE are specifi ed with respect to consistent belief change extensions. Dropping the consistency condition in EXT would result in a trivial decision problem because, according to Defi nition 2.1, any belief change scenario always possesses at least one belief change extension. Concerning CHOICE, although here, as well as for EXT, we are primarily interested in consistent belief change extensions, later we relax this condition and deal also with the inconsistent case. For SKEPTICAL, however, the consistency requirement is actually irrelevant, because it holds that a formula $\phi$ is contained in all belief change extensions of a given belief change scenario $B$ iff it is contained in all consistent belief change extensions of $B$. In general, EXT is arguably less interesting than CHOICE or SKEPTICAL, given that it depends only on the consistency of the constituents of the given belief change scenario-however, the relevance of this task lies in the corresponding search problem, i.e., in the actual computation of all consistent belief change extensions.

\subsection{Encodings of the Basic Tasks}

From now on we assume that, for any belief change scenario $B=(K, R, C)$, its constituents $K, R$, and $C$ are fi nite; thus, these sets are also represented as the conjunction of their elements. Furthermore, for our subsequent encodings it is convenient to use the following alternative characterisation of belief change extensions, which is a straightforward consequence of results due to Delgrande and Schaub [12]. Basically, this characterisation shows that sets of equivalences can be restricted to subsets of $\left\{p \equiv p^{\prime} \mid p \in \operatorname{Var}(B)\right\}$.

Proposition 4.1 For any belief change scenario $B=(K, R, C)$ in $\mathcal{L}_{\mathcal{P}}$, there is a one-to-one correspondence between the determining sets $E Q \subseteq\left\{p \equiv p^{\prime} \mid p \in \mathcal{P}\right\}$ of the belief change extensions of $B$ and sets $E Q^{\sharp} \subseteq\left\{p \equiv p^{\prime} \mid p \in \operatorname{Var}(B)\right\}$ satisfying the following conditions:

(a) $K^{\prime} \cup E Q^{\sharp} \cup R \cup C \nvdash \perp$; and 
(b) for each $p \in \operatorname{Var}(B)$ with $\left(p \equiv p^{\prime}\right) \notin E Q^{\sharp}$, we have $K^{\prime} \cup E Q^{\sharp} \cup\left\{p \equiv p^{\prime}\right\} \cup R \cup C \vdash \perp$.

In particular, for belief change extension $E$ of $B$ with determining set $E Q \subseteq\left\{p \equiv p^{\prime} \mid p \in \mathcal{P}\right\}$, it holds that $E=C n\left(K^{\prime} \cup E Q^{\sharp} \cup R\right) \cap \mathcal{L}_{\mathcal{P}}$, where $E Q^{\sharp}$ satisfies Conditions $(a)$ and $(b)$, and $E Q^{\sharp}=E Q \cap\left\{p \equiv p^{\prime} \mid p \in \operatorname{Var}(B)\right\}$.

Thus, for a belief change extension $E=C n\left(K^{\prime} \cup E Q \cup R\right) \cap \mathcal{L}_{\mathcal{P}}$ with $E Q \subseteq\left\{p \equiv p^{\prime} \mid p \in\right.$ $\mathcal{P}\}$, we also refer, with a slight abuse of notation, to a set of form $E Q^{\sharp} \subseteq\left\{p \equiv p^{\prime} \mid p \in \operatorname{Var}(B)\right\}$, satisfying the above Conditions (a) and (b) and corresponding to $E Q$, as a determining set of $E$.

We proceed with the following basic QBF module:

Definition 4.1 Let $B=(K, R, C)$ be a belief change scenario over $\mathcal{L}_{\mathcal{P}}$, let $V=\operatorname{Var}(B)$ be the set of variables occurring in $B$, and let $V_{e q}=\left\{p_{e q} \mid p \in V\right\}$ be a set of new variables. Then,

$$
\mathcal{M}[B]=K^{\prime} \wedge\left(V_{e q} \leq\left(V \equiv V^{\prime}\right)\right) \wedge R .
$$

The computation of belief change extensions can be expressed in terms of QBFs as follows:

Theorem 4.2 Let $B=(K, R, C)$ be a belief change scenario in $\mathcal{L}_{\mathcal{P}}$, let $V=\operatorname{Var}(B)$ be the atoms occurring in $B$, and let $V_{e q}=\left\{p_{e q} \mid p \in V\right\}$ be a set of variables disjoint from $V$ and $V^{\prime}$. Furthermore, let $E Q \subseteq\left\{p \equiv p^{\prime} \mid p \in \operatorname{Var}(B)\right\}$ be a set of equivalences and let $M \subseteq V_{e q}$ be defined such that $p_{\text {eq }} \in M$ iff $\left(p \equiv p^{\prime}\right) \in E Q$.

Then, $C n\left(K^{\prime} \cup E Q \cup R\right) \cap \mathcal{L}_{\mathcal{P}}$ is a belief change extension of $B$ iff $M$ is a model of the $Q B F$

$$
\begin{aligned}
\mathcal{T}_{\text {ext }}[B]= & \exists V \exists V^{\prime}(\mathcal{M}[B] \wedge C) \wedge \\
& \bigwedge_{p \in V}\left(\neg p_{e q} \rightarrow \neg \exists V \exists V^{\prime}\left(\left(p \equiv p^{\prime}\right) \wedge \mathcal{M}[B] \wedge C\right)\right) .
\end{aligned}
$$

Note that $V_{e q}$ constitutes the set of free variables of $\mathcal{T}_{\text {ext }}[B]$. Intuitively, $V_{\text {eq }}$ guesses a set $E Q$ of equivalences determining a belief change extension of $B$. The first conjunct of $\mathcal{T}_{\text {ext }}[B]$ checks consistency, and the second conjunct checks whether $E Q$ is maximal with respect to set containment.

We remark that in order to encode the computation of belief change extensions according to their original formulation [12], wherein members of $C$ are individually consistent with respect to a belief change extension, $\mathcal{T}_{\text {ext }}[B]$ is modifi ed in the following way:

$$
\begin{aligned}
\bigwedge_{\psi \in C \cup\{\perp\}}\left(\exists V \exists V^{\prime}(\mathcal{M}[B] \wedge \neg \psi)\right) \wedge \\
\bigwedge_{p \in V}\left[\neg p_{e q} \rightarrow \neg \bigwedge_{\psi \in C \cup\{\perp\}}\left(\exists V \exists V^{\prime}\left(\left(p \equiv p^{\prime}\right) \wedge \mathcal{M}[B] \wedge \neg \psi\right)\right)\right] .
\end{aligned}
$$

For an illustration of the translation $\mathcal{T}_{\text {ext }}[\cdot]$, consider the belief change scenario $B=(\{p \wedge$ $q\},\{\neg p \vee \neg q\}, \emptyset)$ from Section 2.4. The free variables of $\mathcal{T}_{\text {ext }}[B]$ are given by $\left\{p_{e q}, q_{e q}\right\}$, so we get the following four interpretations serving as potential models of $\mathcal{T}_{\text {ext }}[B]$ :

$$
\begin{array}{ll}
M_{1}=\{\} ; & M_{3}=\left\{q_{e q}\right\} \\
M_{2}=\left\{p_{e q}\right\} ; & M_{4}=\left\{p_{e q}, q_{e q}\right\} .
\end{array}
$$


Since $B$ has two belief change extensions, generated by $E Q_{1}=\left\{p \equiv p^{\prime}\right\}$ and $E Q_{2}=\left\{q \equiv q^{\prime}\right\}$ (cf. Table 1), we expect $M_{2}$ and $M_{3}$ to be models of $\mathcal{T}_{\text {ext }}[B]$. Let us fi rst look at the left conjunct, $\exists V \exists V^{\prime}(\mathcal{M}[B] \wedge C)$, of $\mathcal{T}_{\text {ext }}[B]$. For $B$ as above, we obtain

$$
\begin{aligned}
& \exists V \exists V^{\prime}(\mathcal{M}[B] \wedge C)= \\
& \quad \exists p q p^{\prime} q^{\prime}\left(\left(p^{\prime} \wedge q^{\prime}\right) \wedge\left(p_{e q} \rightarrow\left(p \equiv p^{\prime}\right)\right) \wedge\left(q_{e q} \rightarrow\left(q \equiv q^{\prime}\right)\right) \wedge(\neg p \vee \neg q)\right) .
\end{aligned}
$$

This QBF has three models, viz. $M_{1}, M_{2}$, and $M_{3}$. Interpretation $M_{1}$ is a model because both conjuncts $\left(p_{e q} \rightarrow\left(p \equiv p^{\prime}\right)\right)$ and $\left(q_{e q} \rightarrow\left(q \equiv q^{\prime}\right)\right)$ of (3) evaluate to true (given that $p_{e q}, q_{e q} \notin$ $\left.M_{1}\right)$, and since the remaining formula $\left(p^{\prime} \wedge q^{\prime}\right) \wedge(\neg p \vee \neg q)$ is consistent. For $M_{2}$, we similarly get that $\left(q_{e q} \rightarrow\left(q \equiv q^{\prime}\right)\right)$ is true and that $\left(p^{\prime} \wedge q^{\prime}\right) \wedge\left(p_{e q} \rightarrow\left(p \equiv p^{\prime}\right)\right) \wedge(\neg p \vee \neg q)$ is consistent, since $\left\{p, p^{\prime}, q^{\prime}\right\}$ is a model of $\left(p^{\prime} \wedge q^{\prime}\right) \wedge\left(p \equiv p^{\prime}\right) \wedge(\neg p \vee \neg q) . M_{3}$ is a model by analogous arguments. However, $M_{4}$ is not a model of (3). This is because, under $M_{4}$, the propositional part of (3) can be reduced to

$$
\left(p^{\prime} \wedge q^{\prime}\right) \wedge\left(p \equiv p^{\prime}\right) \wedge\left(q \equiv q^{\prime}\right) \wedge(\neg p \vee \neg q),
$$

which is not satisfi able.

Hence, only $M_{1}, M_{2}$, or $M_{3}$ are possible models of $\mathcal{T}_{\text {ext }}[B]$.

Now we investigate the remaining conjuncts of $\mathcal{T}_{\text {ext }}[B]$, which are given by

$$
\begin{gathered}
\Phi_{1}=\left[\neg p _ { e q } \rightarrow \neg \exists p q p ^ { \prime } q ^ { \prime } \left(\left(p \equiv p^{\prime}\right) \wedge\left(p^{\prime} \wedge q^{\prime}\right) \wedge\left(p_{e q} \rightarrow\left(p \equiv p^{\prime}\right)\right) \wedge\right.\right. \\
\left.\left.\wedge\left(q_{e q} \rightarrow\left(q \equiv q^{\prime}\right)\right) \wedge(\neg p \vee \neg q)\right)\right]
\end{gathered}
$$

and

$$
\begin{gathered}
\Phi_{2}=\left[\neg q _ { e q } \rightarrow \neg \exists p q p ^ { \prime } q ^ { \prime } \left(\left(q \equiv q^{\prime}\right) \wedge\left(p^{\prime} \wedge q^{\prime}\right) \wedge\left(p_{e q} \rightarrow\left(p \equiv p^{\prime}\right)\right) \wedge\right.\right. \\
\left.\left.\wedge\left(q_{e q} \rightarrow\left(q \equiv q^{\prime}\right)\right) \wedge(\neg p \vee \neg q)\right)\right] .
\end{gathered}
$$

First, consider interpretation $M_{2}$. Given that $p_{e q} \in M_{2}$, conjunct $\Phi_{1}$ evaluates to true, and it remains to analyse $\Phi_{2}$. The latter formula evaluates to true if

$$
\left(q \equiv q^{\prime}\right) \wedge\left(p^{\prime} \wedge q^{\prime}\right) \wedge\left(p_{e q} \rightarrow\left(p \equiv p^{\prime}\right)\right) \wedge\left(q_{e q} \rightarrow\left(q \equiv q^{\prime}\right)\right) \wedge(\neg p \vee \neg q)
$$

is not satisfi able. However, given $M_{2}$, (5) reduces to (4), which is indeed unsatisfi able. Hence, $M_{2}$ is a model of $\Phi_{2}$, and thus also a model of $\mathcal{T}_{\text {ext }}[B]$. By a similar argument it follows that $M_{3}$ is a model of $\Phi_{1} \wedge \Phi_{2}$. It remains to see that $M_{1}$ is not a model of $\Phi_{1} \wedge \Phi_{2}$. In fact, it holds that $\nu_{M_{1}}\left(\Phi_{1}\right)=\nu_{M_{1}}\left(\Phi_{2}\right)=0$. We show the case of $\Phi_{1}$ (the case of $\Phi_{2}$ follows analogously). Since $M_{1}=\{\}, \Phi_{1}$ is false under $M_{1}$ iff

$$
\exists p q p^{\prime} q^{\prime}\left(\left(p \equiv p^{\prime}\right) \wedge\left(p^{\prime} \wedge q^{\prime}\right) \wedge\left(p_{e q} \rightarrow\left(p \equiv p^{\prime}\right)\right) \wedge\left(q_{e q} \rightarrow\left(q \equiv q^{\prime}\right)\right) \wedge(\neg p \vee \neg q)\right)
$$

is true under $M_{1}$. Given that both $p_{e q}$ and $q_{e q}$ are false under $M_{1}$, the previous condition holds iff

$$
\left(p \equiv p^{\prime}\right) \wedge\left(p^{\prime} \wedge q^{\prime}\right) \wedge(\neg p \vee \neg q)
$$


is satisfi able. Clearly, this is the case, since $\left\{p, p^{\prime}, q^{\prime}\right\}$ is a satisfying truth assignment for (6). Thus, $M_{1}$ is not a model of $\Phi_{1}$. This shows that $M_{1}$ is not a model of $\mathcal{T}_{\text {ext }}[B]$.

Concerning a QBF encoding for EXT, it immediately follows from Theorem 4.2 that a belief change scenario $B=(K, R, C)$ with $V=\operatorname{Var}(B)$ has a consistent belief change extension iff $\exists V_{\text {eq }} \mathcal{T}_{\text {ext }}[B]$ evaluates to true. However, this encoding is in some sense not optimal because it is possible to characterise EXT in terms of a simpler QBF, corresponding to an ordinary satisfi ability problem, by observing that $B$ has a consistent belief change extension iff $K^{\prime} \cup R \cup C$ is consistent. Consequently, we can state the following result:

Theorem 4.3 Let $B=(K, R, C)$ be a belief change scenario in $\mathcal{L}_{\mathcal{P}}$ and let $V=\operatorname{Var}(B)$.

Then, $B$ has a consistent belief change extension iff $\exists V \exists V^{\prime}\left(K^{\prime} \wedge R \wedge C\right)$ evaluates to true.

Next, we discuss the translations of the reasoning tasks CHOICE and SKEPTICAL. We begin with the encodings of the corresponding search problems.

Theorem 4.4 Let $B=(K, R, C)$ be a belief change scenario in $\mathcal{L}_{\mathcal{P}}$ and let $\phi$ be a formula. Furthermore, let $V=\operatorname{Var}(B)$, let $W=\operatorname{Var}(B) \cup \operatorname{Var}(\phi)$, and let $V_{\text {eq }}=\left\{p_{\text {eq }} \mid p \in V\right\}$ be a set of globally new variables. Finally, consider the $Q B F$

$$
\mathcal{M}[B]=K^{\prime} \wedge\left(V_{e q} \leq\left(V \equiv V^{\prime}\right)\right) \wedge R
$$

from Definition 4.1.

Then, for $E Q \subseteq\left\{p \equiv p^{\prime} \mid p \in \operatorname{Var}(B)\right\}$ and $M \subseteq V_{\text {eq }}$ such that $\left(p \equiv p^{\prime}\right) \in E Q$ iff $p_{\text {eq }} \in M$, the following properties hold:

1. $C n\left(K^{\prime} \cup E Q \cup R\right) \cap \mathcal{L}_{\mathcal{P}}$ is a belief change extension of $B$ containing $\phi$ iff $M$ is a model of the $Q B F$

$$
\mathcal{T}_{\text {choice }}[B, \phi]=\mathcal{T}_{\text {ext }}[B] \wedge \forall W\left(\left(\exists V^{\prime} \mathcal{M}[B]\right) \rightarrow \phi\right)
$$

2. $C n\left(K^{\prime} \cup E Q \cup R\right) \cap \mathcal{L}_{\mathcal{P}}$ is a belief change extension of $B$ not containing $\phi$ iff $M$ is a model of the $Q B F$

$$
\mathcal{T}_{\text {skept }}[B, \phi]=\mathcal{T}_{\text {ext }}[B] \wedge \neg \forall W\left(\left(\exists V^{\prime} \mathcal{M}[B]\right) \rightarrow \phi\right)
$$

Intuitively, the two encodings $\mathcal{T}_{\text {choice }}[B, \phi]$ and $\mathcal{T}_{\text {skept }}[B, \phi]$ are realised by (i) checking whether a selected set of equivalences determines a consistent belief change extension $E$ of $B$, and (ii) checking whether $E$ contains a given formula $\phi$, or checking whether $E$ does not contain $\phi$. Task (i) is modeled using the basic encoding $\mathcal{T}_{\text {ext }}[B]$, and Task (ii) is captured by a suitable QBF expressing derivability (in case of $\mathcal{T}_{\text {choice }}[B, \phi]$ ) or non-derivability (in case of $\mathcal{T}_{\text {skept }}[B, \phi]$ ) of $\phi$ from the selected belief change extension. Observe that the selection process is facilitated in terms of the members from $V_{\text {eq }}$, which represent the free variables of $\mathcal{T}_{\text {choice }}[B, \phi]$ and $\mathcal{T}_{\text {skept }}[B, \phi]$.

Concerning the decision problems CHOICE and SKEPTICAL, QBF encodings for these tasks are obtained from $\mathcal{T}_{\text {choice }}[B, \phi]$ and $\mathcal{T}_{\text {skept }}[B, \phi]$ as follows. CHOICE is expressed by the closed QBF $\exists V_{e q} \mathcal{T}_{\text {choice }}[B, \phi]$, which states that there is some some set $M \subseteq V_{e q}$ corresponding to a set $E Q$ 
of equivalences such that $E Q$ determines a consistent belief change extension $E$ entailing $\phi$, and SKEPTICAL is realised by the closed QBF $\neg \exists V_{\text {eq }} \mathcal{T}_{\text {skept }}[B, \phi]$, which expresses that there is no set $M \subseteq V_{e q}$ corresponding to a set $E Q$ of equivalences such that $E Q$ determines a consistent belief change extension $E$ not entailing $\phi$. Thus, we obtain the following corollary:

Corollary 4.5 Let $B$ be a belief change scenario and $\phi$ a formula. Then,

1. $\phi$ is contained in at least one consistent belief change extension of $B$ iff $\exists V_{\text {eq }} \mathcal{T}_{\text {choice }}[B, \phi]$ evaluates to true, and

2. $\phi$ is contained in all belief change extensions of $B$ iff $\neg \exists V_{\text {eq }} \mathcal{T}_{\text {skept }}[B, \phi]$ evaluates to true.

In contrast to task EXT, which can be expressed by a QBF containing only one sort of quantifi er, here we obtain encodings possessing both existential quantifiers as well as universal ones. As we show in Section 5, this quantifi er alternation is in some sense unavoidable and reflects the inherent complexity of CHOICE and SKEPTICAL.

We remark that discarding the consistency condition of CHOICE can be easily incorporated into the translation $\exists V_{e q} \mathcal{T}_{\text {choice }}[\cdot, \cdot]$. Indeed, it is a simple matter to check that there is a (possibly inconsistent) belief change extension of $B=(K, R, C)$ containing formula $\phi$ iff the QBF

$$
\left(\exists V \exists V^{\prime}\left(K^{\prime} \wedge R \wedge C\right)\right) \rightarrow\left(\exists V_{\text {eq }} \mathcal{T}_{\text {choice }}[B, \phi]\right)
$$

evaluates to true.

Finally, observe that Theorems 4.2, 4.3, and 4.4, as well as Corollary 4.5, provide encodings of reasoning tasks for arbitrary belief change scenarios. In particular, they subsume the characterisation of the corresponding reasoning tasks associated with revision and contraction, as illustrated by the revision example discussed previously. For convenience, we list the tasks for revision:

REXT: Given a knowledge base $K$ and some formula $\alpha$, decide whether a consistent belief change extension of $B=(K,\{\alpha\}, \emptyset)$ exists.

RCHOICE: Given a knowledge base $K$ and formulas $\alpha$ and $\phi$, decide whether there is some consistent choice revision $K \dot{+}_{c} \alpha$ containing $\phi$.

RSKEPTICAL: Given a knowledge base $K$ and formulas $\alpha$ and $\phi$, decide whether $\phi$ is contained in the skeptical revision $K \dot{+} \alpha$.

The corresponding tasks for belief contraction, denoted by CEXT, CCHOICE, and CSKEPTICAL, are defi ned accordingly.

\subsection{Expressing Changed Knowledge Bases}

Another interesting issue in the context of belief change is to determine the actual form of a given knowledge base after a revision or contraction operation has been applied to it. This task has already been analysed by Delgrande and Schaub [12], and, as we show in the following, it can also be described in terms of QBFs. Before going into details, we briefly summarise the relevant previous results [12], starting with some notation. 
Given a belief change scenario $B$ in language $\mathcal{L}_{\mathcal{P}}$ along with a set of equivalences $E Q_{i} \subseteq\{p \equiv$ $\left.p^{\prime} \mid p \in \mathcal{P}\right\}$, defi ne

$$
\begin{aligned}
& \mathcal{P}_{E Q_{i}}=\left\{p \in \mathcal{P} \mid p \equiv p^{\prime} \in E Q_{i}\right\}, \quad \text { and } \\
& \mathcal{P}_{\overline{E Q_{i}}}=\mathcal{P} \backslash \mathcal{P}_{E Q_{i}} .
\end{aligned}
$$

Then, for $\phi \in \mathcal{L}_{\mathcal{P}}$, let $\lceil\phi\rceil_{i}$ be the result of replacing in $\phi$ each $p \in \mathcal{P}_{\overline{E Q}_{i}}$ by $\neg p$. Furthermore, for a set of functions

$$
\Pi_{i}=\left\{\pi_{i}^{k} \mid \pi_{i}^{k}: \mathcal{P}_{\overline{E Q}_{i}} \rightarrow\{\top, \perp\}\right\}
$$

let $\lfloor\phi\rfloor_{i}^{k}$ be the result of replacing in $\phi$ each $p \in \mathcal{P}_{\overline{E Q}}$ by $\pi_{i}^{k}(p)$.

Then, the result of applying revision or contraction to a given knowledge base is described by the following formulas:

Proposition 4.6 ([12]) Let $K$ be a finite knowledge base and $\alpha$ some formula. Then,

1. $K \dot{+} \alpha$ is logically equivalent to $\bigvee_{i \in I}\lceil K\rceil_{i} \wedge \alpha$, and

2. $K \dot{-} \alpha$ is logically equivalent to $\bigvee_{i \in I, \pi_{i}^{k} \in \Pi_{i}}\lfloor K\rfloor_{i}^{k}$,

for $\left(E Q_{i}\right)_{i \in I}$ being the family of sets of equivalences determining the belief change extensions of $(K,\{\alpha\}, \emptyset)$ and $(K, \emptyset,\{\neg \alpha\})$, respectively. tion.

We can express the models of revision and contraction by QBFs using the following construc-

Definition 4.2 Let $B$ be a belief change scenario in $\mathcal{L}_{\mathcal{P}}$ with $V=\operatorname{Var}(B)$, and let $V_{\text {eq }}=\left\{p_{\text {eq }} \mid\right.$ $p \in \operatorname{Var}(B)\}$ be a set of new variables. Then,

$$
\mathcal{T}_{m}[B]=\exists V_{\text {eq }}\left(\mathcal{T}_{\text {ext }}[B] \wedge \exists V^{\prime} \mathcal{M}[B]\right) .
$$

Observe that $\mathcal{T}_{m}[B]$ is an open QBF having $V$ as its set of free variables. We obtain the following result:

Theorem 4.7 Let $K$ be a finite knowledge base, $\alpha$ some formula, and $V=\operatorname{Var}(K \cup\{\alpha\})$. Then,

1. $M \subseteq V$ is a model of $K \dot{+} \alpha$ iff $M$ is a model of $\mathcal{T}_{m}[(K,\{\alpha\}, \emptyset)]$, and

2. $M \subseteq V$ is a model of $K \dot{-} \alpha$ iff $M$ is a model of $\mathcal{T}_{m}[(K, \emptyset,\{\neg \alpha\})]$.

By collecting all the models of $K \dot{+} \alpha$ or $K \dot{-} \alpha$ into a single formula, we thus obtain a disjunctive normal form of $K$ after revision or contraction with $\alpha$.

To formally express this, given a belief change scenario $B$ with $V=\operatorname{Var}(B)$, defi ne

$$
F[B]=\bigvee_{M \in \operatorname{Mod}\left(\mathcal{T}_{m}[B]\right)}\left(M \wedge \bigwedge_{p \in(V \backslash M)} \neg p\right) .
$$

Then, we get the following characterisation, representing an alternative to Proposition 4.6: 
Corollary 4.8 Let $K$ and $\alpha$ be as in Theorem 4.7. Then,

1. $K \dot{+} \alpha$ is logically equivalent to $F[(K,\{\alpha\}, \emptyset)]$, and

2. $K \dot{-} \alpha$ is logically equivalent to $F[(K, \emptyset,\{\neg \alpha\})]$.

For illustration of transformation $\mathcal{T}_{m}[\cdot]$, consider again the belief change scenario $B=$ $(\{p \wedge q\},\{\neg p \vee \neg q\}, \emptyset)$. As already discussed in Section 2.4, B possesses the skeptical revision $C n(\{p \equiv \neg q\})$, which can be rewritten as $C n(\{(p \wedge \neg q) \vee(\neg p \wedge q)\})$. Thus, in virtue of Theorem 4.7, we expect $\{p\}$ and $\{q\}$ to be the models of

$$
\mathcal{T}_{m}[B]=\exists V_{e q}\left(\mathcal{T}_{\text {ext }}[B] \wedge \exists V^{\prime} \mathcal{M}[B]\right) .
$$

We fir rst compute the models of the QBF

$$
\mathcal{T}_{\text {ext }}[B] \wedge \exists V^{\prime} \mathcal{M}[B] .
$$

The free variables of (7) are given by $V_{e q} \cup V=\left\{p_{\text {eq }}, q_{e q}\right\} \cup\{p, q\}$. As argued previously, $\mathcal{T}_{\text {ext }}[B]$ is true under interpretations $\left\{p_{\text {eq }}\right\}$ and $\left\{q_{\text {eq }}\right\}$. In fact, since $\mathcal{T}_{\text {ext }}[B]$ has no free variables from $V$, it holds that $\left\{p_{e q}\right\} \cup U$ and $\left\{q_{e q}\right\} \cup U$ are also models of $\mathcal{T}_{\text {ext }}[B]$, for any set $U \subseteq V$. Consider now the second conjunct of (7), which is given by

$$
\exists p^{\prime} q^{\prime}\left(\left(p^{\prime} \wedge q^{\prime}\right) \wedge\left(p_{e q} \rightarrow\left(p \equiv p^{\prime}\right)\right) \wedge\left(q_{e q} \rightarrow\left(q \equiv q^{\prime}\right)\right) \wedge(\neg p \vee \neg q)\right)
$$

This formula is obviously true under the following interpretations:

$$
M_{1}=\left\{p_{e q}, p\right\}, \quad M_{2}=\left\{q_{e q}, q\right\},
$$

as well as under every interpretation in which both $p_{e q}$ and $q_{e q}$ are false. Thus, only $M_{1}$ and $M_{2}$ satisfy both conjuncts of (7). Taking the existential closure of QBF (7) with respect to the variables $p_{e q}$ and $q_{e q}$, we get that $\{p\}$ and $\{q\}$ are the only models of $\mathcal{T}_{m}[B]$. Accordingly, we get $F[B]=(p \wedge \neg q) \vee(\neg p \wedge q)$.

\section{Complexity Results}

In this section, we analyse the computational complexity of the tasks considered so far. Additionally, we also deal with the complexity of checking whether a given set of equivalences determines some consistent belief change extension of a given belief change scenario.

A particular advantage of our reduction approach is that upper complexity bounds are derived directly from the respective QBF encodings. This is due to the fact that our QBF translations are polynomial in the size of a given belief change scenario, and that the complexity of evaluating a given $\mathrm{QBF} \Phi$ is determined by the quantifi er order of $\Phi$. For each of the upper bounds obtained in this fashion, we show also that they are strict, i.e., they possess a matching lower bound. The results presented here strengthen a previous complexity analysis given by Delgrande and Schaub [11]. 
In what follows, we assume that the reader is familiar with the basic concepts of complexity theory (see, e.g., [40]). For convenience, we briefly recapitulate the defi nitions and some elementary properties of the complexity classes considered in the following. As usual, for any complexity class $C$, by co- $C$ we understand the class of all problems which are complementary to the problems in $C$.

Four complexity classes are relevant here, viz. NP, $\mathrm{D}^{P}, \Sigma_{2}^{P}$, and $\Pi_{2}^{P}$. The class NP consists of all decision problems which can be solved with a nondeterministic Turing machine working in polynomial time; $\mathrm{D}^{P}$ is defi ned as the class of all problems which can be described as the conjunction of two (independent) problems from NP and co-NP; $\Sigma_{2}^{P}$ is the class of all problems solvable on a nondeterministic Turing machine in polynomial time having access to an oracle for problems in NP; fi nally, $\Pi_{2}^{P}=\mathrm{co}-\Sigma_{2}^{P}$.

Observe that NP, $\Sigma_{2}^{P}$, and $\Pi_{2}^{P}$ are part of the polynomial hierarchy, which is given by the following sequence of objects: the initial elements are

$$
\Delta_{0}^{P}=\Sigma_{0}^{P}=\Pi_{0}^{P}=\mathrm{P}
$$

and, for $i>0$,

$$
\Delta_{i}^{P}=\mathrm{P}^{\Sigma_{i-1}^{P}}, \quad \Sigma_{i}^{P}=\mathrm{NP}^{\Sigma_{i-1}^{P}}, \quad \text { and } \quad \Pi_{i}^{P}=\mathrm{co-} \mathrm{NP}^{\Sigma_{i-1}^{P}}
$$

Here, $\mathrm{P}$ is the class of all problems solvable on a deterministic Turing machine in polynomial time, and, for complexity classes $C$ and $A$, by $C^{A}$ we understand the relativised version of $C$, consisting of all problems which can be decided by Turing machines of the same sort and time bound as in $C$, only that the machines have access to an oracle for problems in $A$. It holds that $\Sigma_{1}^{P}=\mathrm{NP}$, $\Sigma_{2}^{P}=\mathrm{NP}^{\mathrm{NP}}$, and $\Pi_{2}^{P}=\mathrm{co}-\mathrm{NP}^{\mathrm{NP}}$. A problem is said to be at the $k$-th level of the polynomial hierarchy iff it is in $\Delta_{k+1}^{P}$ and either $\Sigma_{k}^{P}$-hard or $\Pi_{k}^{P}$-hard.

The class $\mathrm{D}^{P}$ is part of a family of complexity classes $\mathrm{D}_{k}^{P}, k \geq 1$, where $\mathrm{D}_{1}^{P}=\mathrm{D}^{P}$ and each $\mathrm{D}_{k}^{P}$ consists of all problems expressible as the conjunction of a problem in $\Sigma_{k}^{P}$ and a problem in $\Pi_{k}^{P}$. Notice that, for all $k \geq 1, \Sigma_{k}^{P} \subseteq \mathrm{D}_{k}^{P} \subseteq \Sigma_{k+1}^{P}$ holds; in fact, both inclusions are widely conjectured to be strict. Moreover, any problem in $\mathrm{D}_{k}^{P}$ can be solved with two $\Sigma_{k}^{P}$ oracle calls, and is thus intuitively easier than a problem complete for $\Delta_{k}^{P}$.

In the same way as the satisfi ability problem of classical propositional logic is the "prototypical" problem of NP, i.e., being an NP-complete problem, the satisfi ability problem of QBFs possessing $k-1$ quantifi er alternations is the "prototypical" problem of the $k$-th level of the polynomial hierarchy. More specifi cally, the following property holds:

Proposition 5.1 ([59]) Given a propositional formula $\phi$ whose atoms are partitioned into $i \geq 1$ sets $V_{1}, \ldots, V_{i}$, deciding whether $\exists V_{1} \forall V_{2} \exists V_{3} \ldots \mathrm{Q} V_{i} \phi$ evaluates to true is $\Sigma_{i}^{P}$-complete, where $\mathrm{Q}=\exists$ if $i$ is odd and $\mathrm{Q}=\forall$ if $i$ is even. Moreover, the problem remains $\Sigma_{i}^{P}$-hard even if $\phi$ is in conjunctive normal form and $i$ is odd, or if $\phi$ is in disjunctive normal form and $i$ is even.

From this result it follows that the evaluation problem of QBFs of form $\forall V_{1} \exists V_{2} \forall V_{3} \ldots \mathrm{Q} V_{i} \phi$ is $\Pi_{i}^{P}$-complete, where $\mathrm{Q}=\forall$ if $i$ is odd and $\mathrm{Q}=\exists$ if $i$ is even. As well, the problem remains $\Pi_{i}^{P}$-hard even if $\phi$ is in disjunctive normal form and $i$ is odd, or if $\phi$ is in conjunctive normal form and $i$ is even. 
Given the above characterisations, we can estimate upper complexity bounds for the decision problems discussed in Section 4 by simply inspecting the quantifi er order of the respective QBF encodings. This can be argued as follows. First of all, by applying the transformation rules described in Proposition 3.2, each of the above QBF encodings can be transformed in polynomial time into a closed QBF in prenex form. Then, by invoking Proposition 5.1 and observing that completeness of a decision problem $D$ for a complexity class $C$ implies membership of $D$ in $C$, the quantifi er order of the resultant QBFs determines in which class of the polynomial hierarchy the corresponding decision problem lies.

Applying this method to the decision problems EXT, CHOICE, and SKEPTICAL, we get the following results. To begin with, according to Theorem 4.3, we have that EXT lies in NP. Hence, REXT and CEXT are also in NP because they are just special cases of EXT. Furthermore, the encoding $\exists V_{e q} \mathcal{T}_{\text {choice }}[B, \phi]$ for CHOICE can be transformed into a QBF of prenex form $\exists W_{1} \forall W_{2} \psi$, and, dually, the encoding $\neg \exists V_{\text {eq }} \mathcal{T}_{\text {skept }}[B, \phi]$ for SKEPTICAL can be transformed into a QBF of prenex form $\forall Z_{1} \exists Z_{2} \varphi$, where both $\psi$ and $\varphi$ are purely propositional. Thus, CHOICE is in $\Sigma_{2}^{P}$, and SKEPTICAL is in $\Pi_{2}^{P}$. Similar to the case of EXT, $\Sigma_{2}^{P}$ is also an upper bound for RCHOICE and CCHOICE, and $\Pi_{2}^{P}$ is an upper bound for RSKEPTICAL and CSKEPTICAL.

Concerning lower complexity bounds, it turns out that all of the above given estimations are strict, i.e., the considered decision problems are hard for the respective complexity classes. Summarising, we can state the following results:

Theorem 5.2 The decision problems EXT, CHOICE, and SKEPTICAL, as well as its variants for revision and contraction, enjoy the following completeness properties:

1. EXT, REXT, and CEXT are NP-complete;

2. CHOICE, RCHOICE, and CCHOICE are $\Sigma_{2}^{P}$-complete; and

3. SKEPTICAL, RSKEPTICAL, and CSKEPTICAL are $\Pi_{2}^{P}$-complete.

Thus, the completeness results for CHOICE and SKEPTICAL, as well as for their specialisations for revision and contraction, imply that, unless the polynomial hierarchy collapses, it is not possible to effi ciently represent these tasks in terms of QBFs having a prenex form with only one sort of quantifi er, i.e., these tasks cannot be polynomially reduced to standard propositional logic. Hence, under the above proviso, the encodings described in Corollary 4.5 cannot be simplifi ed further to avoid an inherent quantifi er alternation.

Rounding off our complexity analysis, we deal with the problem of checking whether a given set of equivalences determines some consistent belief change extension of a given belief change scenario.

Theorem 5.3 Given a belief change scenario $B$ and a set $E Q \subseteq\left\{p \equiv p^{\prime} \mid p \in \operatorname{Var}(B)\right\}$, checking whether $E Q$ determines some consistent belief change extension of $B$ is $\mathrm{D}^{P}$-complete.

\section{Implementation}

Our methodology for expressing reasoning tasks associated with belief change scenarios in terms of quantifi ed Boolean formulas is motivated by the availability of several practicably effi cient QBFsolvers. Among the different tools, there is a propositional theorem-prover, boole, based on 


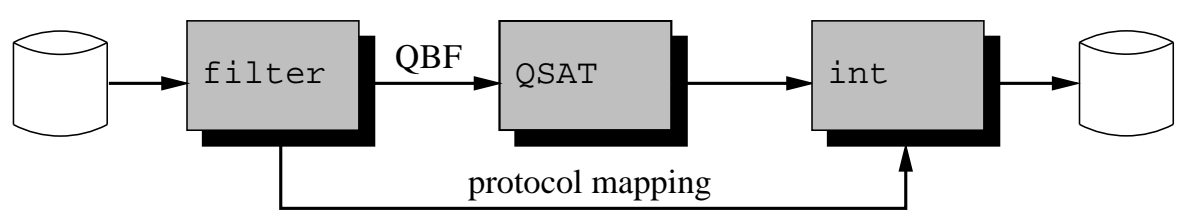

Figure 1: Architecture to use different QBF-solvers.

binary decision diagrams, ${ }^{3}$ a system using a generalised resolution principle [27], several provers implementing an extended Davis-Putnam procedure [4, 46, 19, 24, 30], as well as a distributed algorithm running on a PC-cluster [19].

The translations discussed in the previous section have been implemented as a special module of the reasoning system QUIP [15, 14, 41, 17], a prototype tool for solving various nonmonotonic reasoning tasks based on reductions to QBFs. Among others, QUIP handles tasks for logic-based abduction, default logic, several types of modal nonmonotonic logics, and the stable model semantics for logic programs.

The general architecture of QUIP is depicted in Figure 1. QUIP consists of three parts, viz. the filter program, a QBF-evaluator, and the interpreter int. The input fi lter translates the given problem description (in our case, a belief change scenario and a specifi ed reasoning task) into the corresponding quantifi ed Boolean formula, which is then sent to the QBF-evaluator. The current version of QUIP provides interfaces to most of the sequential QBF-solvers mentioned above. For the solvers requiring prenex normal form, the QBFs are translated into structure preserving normal form [13, 42]. The result of the QBF-evaluator is interpreted by int. Depending on the capabilities of the employed QBF-evaluator, int provides an explanation in terms of the underlying problem instance (e.g., listing all consistent defi nitional extensions of a given belief change scenario). This task relies on a protocol mapping of internal variables of the generated QBF into concepts of the problem description which is provided by filter.

The system QUIP has been implemented in C using standard tools like LEX and YACC (comprising a total of 2000 lines of code, excluding the used QBF-solver); it runs currently in a Unix environment (Sun/Solaris and Linux), but is easily portable to other operating systems as well.

Initial tests on a series of randomly generated benchmarks, using the system boole as underlying reasoning engine, showed that the current approach can handle problems built of up to 300 variables within a couple of seconds. As well, on the considered examples, the system outperforms a dedicated ad-hoc implementation [10] realised in JAVA.

\section{Conclusion}

We have shown how belief revision and belief contraction, as defi ned using belief change scenarios, can be axiomatised by means of quantifi ed Boolean formulas. The general mechanism of our approach is to translate (in polynomial time) a reasoning problem, expressed in terms of belief change scenarios, into the evaluation problem for QBFs. Following this, we use a QBF-evaluator to compute the resultant instances.

\footnotetext{
${ }^{3}$ The system can be downloaded from the Web at http://www. cs. cmu. edu/ modelcheck/bdd. html.
} 
The approach has several benefi ts. First, the given axiomatics provides us with further insight about how belief revision and contraction work within belief change scenarios. As well, this axiomatisation allows us to furnish upper bounds for precise complexity results. Last but not least we obtain a straightforward implementation technique of belief change in belief change scenarios by appeal to the existing system QUIP $[15,14]$. Note that the availability of a parallel QBF-evaluation solver [19] yields also in a direct way a distributed decision procedure for the encoded problems. This convenient situation obviously avoids designing special-purposed distributed algorithms for the problems under consideration.

The implemented operators possess good formal properties, in that most AGM postulates obtain. In particular, the postulate of irrelevance of syntax is retained, and so the results of a belief change operation is independent of the syntactic expression of its arguments. While the interesting decision problems involving reasoning lie at the second level of the polynomial hierarchy, it remains to be seen whether the implementation may nonetheless prove practical for large-scale applications.

\section{A Proofs}

Proof 4.2 Let us write $\mathcal{T}_{\text {ext }}[B]$ as $\Phi_{1} \wedge \Phi_{2}$, where

$$
\begin{aligned}
& \Phi_{1}=\exists V \exists V^{\prime}(\mathcal{M}[B] \wedge C), \quad \text { and } \\
& \left.\Phi_{2}=\bigwedge_{p \in V}\left(\neg p_{e q} \rightarrow \neg \exists V \exists V^{\prime}\left(\left(p \equiv p^{\prime}\right) \wedge \mathcal{M}[B] \wedge C\right)\right)\right) .
\end{aligned}
$$

Consider Conditions (a) and (b) of Proposition 4.1. We show that Condition (a) holds iff $M$ is a model of $\Phi_{1}$, and that (b) holds iff $M$ is a model of $\Phi_{2}$.

To begin with, since, by hypothesis, $E Q$ and $M$ satisfy the condition that $\left(p \equiv p^{\prime}\right) \in E Q$ iff $p_{e q} \in M$, we can apply Proposition 3.1 and obtain that $K^{\prime} \cup E Q \cup R \cup C$ is satisfi able iff $M$ is a model of

$$
\exists V \exists V^{\prime}\left(K^{\prime} \wedge R \wedge C \wedge\left(V_{e q} \leq\left(V \equiv V^{\prime}\right)\right)\right)
$$

which is obviously equivalent to $\Phi_{1}$. It remains to show that Condition (b) holds iff $M$ is a model of $\Phi_{2}$.

Consider some $p \in V$ such that $\left(p \equiv p^{\prime}\right) \notin E Q$ and $K^{\prime} \cup E Q \cup\left\{p \equiv p^{\prime}\right\} \cup R \cup C \vdash \perp$. Invoking Proposition 3.1 again, it follows that the last condition holds exactly if the QBF

$$
\neg \exists V \exists V^{\prime}\left(K^{\prime} \wedge\left(p \equiv p^{\prime}\right) \wedge R \wedge C \wedge\left(V_{e q} \leq\left(V \equiv V^{\prime}\right)\right)\right)
$$

is true under $M$. In general, if we perform this test for each $p \in V$ with $\left(p \equiv p^{\prime}\right) \notin E Q$, we get that Condition (b) is equivalent to the condition that the QBF

$$
\bigwedge_{p \in V,\left(p \equiv p^{\prime}\right) \notin E Q} \neg \exists V \exists V^{\prime}\left(K^{\prime} \wedge\left(p \equiv p^{\prime}\right) \wedge R \wedge C \wedge\left(V_{e q} \leq\left(V \equiv V^{\prime}\right)\right)\right)
$$

is true under $M$. Observing that, for any $p \in V,\left(p \equiv p^{\prime}\right) \in E Q$ iff $p_{e q} \in M$, it follows that $M$ is a model of (8) iff it is a model of

$$
\bigwedge_{p \in V} \neg p_{e q} \rightarrow \neg \exists V \exists V^{\prime}\left(K^{\prime} \wedge\left(p \equiv p^{\prime}\right) \wedge R \wedge C \wedge\left(V_{e q} \leq\left(V \equiv V^{\prime}\right)\right)\right) .
$$


Since (9) is logically equivalent to $\Phi_{2}$, we conclude that Condition (b) holds iff $M$ is a model of $\Phi_{2}$.

Proof 4.4 According to Theorem 4.2, $C n\left(K^{\prime} \cup E Q \cup R\right) \cap \mathcal{L}_{\mathcal{P}}$ is a consistent belief change extension of $B$ iff $M$ is a model of $\mathcal{T}_{\text {ext }}[B]$. Thus, for proving Part 1 of the theorem, it suffi ces to show that the following condition holds:

$(*) \phi \in C n\left(K^{\prime} \cup E Q \cup R\right)$ iff $M$ is a model of $\forall W\left(\left(\exists V^{\prime} \mathcal{M}[B]\right) \rightarrow \phi\right)$.

Furthermore, since $M$ is a model of $\forall W\left(\left(\exists V^{\prime} \mathcal{M}[B]\right) \rightarrow \phi\right)$ precisely if $M$ is not a model of $\neg \forall W\left(\left(\exists V^{\prime} \mathcal{M}[B]\right) \rightarrow \phi\right)$, we get that Condition $(*)$ implies that $\phi \notin C n\left(K^{\prime} \cup E Q \cup R\right)$ iff $M$ is a model of $\neg \forall W\left(\left(\exists V^{\prime} \mathcal{M}[B]\right) \rightarrow \phi\right)$, which in turn proves Part 2 of the theorem. It remains to show that $(*)$ holds.

Since $\phi \in C n\left(K^{\prime} \cup E Q \cup R\right)$ iff $K^{\prime} \cup E Q \cup R \cup\{\neg \phi\}$ is unsatisfi able, Proposition 3.1 implies that $\phi \in C n\left(K^{\prime} \cup E Q \cup R\right)$ iff $M$ is a model of

$$
\neg \exists W \exists V^{\prime}\left(K^{\prime} \wedge R \wedge \neg \phi \wedge\left(V_{e q} \leq\left(V \equiv V^{\prime}\right)\right)\right) .
$$

Given that $\phi$ does not contain any primed atoms, we can rewrite (10) by moving $\neg \phi$ outside the scope of the quantifi cation $\exists V^{\gamma}$, thus obtaining

$$
\neg \exists W\left(\exists W^{\prime}\left(K^{\prime} \wedge R \wedge\left(V_{e q} \leq\left(V \equiv V^{\prime}\right)\right)\right) \wedge \neg \phi\right),
$$

which is in turn equivalent to

$$
\neg \exists W\left(\left(\exists V^{\prime} \mathcal{M}[B]\right) \wedge \neg \phi\right) .
$$

But (11) is clearly equivalent to

$$
\forall W\left(\left(\exists V^{\prime} \mathcal{M}[B]\right) \rightarrow \phi\right),
$$

and therefore we obtain that $\phi \in C n\left(K^{\prime} \cup E Q \cup R\right)$ iff $M$ is a model of (12).

Proof 4.7 Let $K$ be a fi nite knowledge base, $\alpha$ some formula, and $V=\operatorname{Var}(K \cup\{\alpha\})$. Recall that, for any set $E Q$ of equivalences, $\mathcal{P}_{E Q}=\left\{p \in \mathcal{P} \mid p \equiv p^{\prime} \in E Q\right\}$ and $\mathcal{P}_{\overline{E Q}}=\mathcal{P} \backslash \mathcal{P}_{E Q}$.

1. Consider $B=(K,\{\alpha\}, \emptyset)$ in $\mathcal{L}_{\mathcal{P}}$. Suppose that $M \subseteq V$ is a model of $K \dot{+} \alpha$. By Proposition 4.6, there is a consistent belief change extension $E_{i_{0}}=C n\left(K^{\prime} \cup E Q_{i_{0}} \cup\{\alpha\}\right) \cap \mathcal{L}_{\mathcal{P}}$ of $B$ (for some $i_{0} \in I$ ) such that $\lceil K\rceil_{i_{0}} \wedge \alpha$ is true under $M$. Define $M_{1}=M \cap \mathcal{P}_{E Q_{i_{0}}}$ and $M_{2}=M \backslash M_{1}$. By construction of formula $\lceil K\rceil_{i_{0}}$, it follows that $M_{1} \cup \bar{M}_{2}$ is a model of $K$, where $\bar{M}_{2}=\left(V \cap \mathcal{P}_{\overline{E Q}_{i_{0}}}\right) \backslash M$. Hence, a simple renaming yields that $M_{1}^{\prime} \cup \bar{M}_{2}^{\prime}$ is a model of $K^{\prime}$. It follows that $M \cup M_{1}^{\prime} \cup \bar{M}_{2}^{\prime}$ is a model of

$$
K^{\prime} \wedge \bigwedge_{p \equiv p^{\prime} \in E Q_{i_{0}}}\left(p \equiv p^{\prime}\right) \wedge \alpha
$$

By setting $J=\left\{p_{e q} \mid p \equiv p^{\prime} \in E Q_{i_{0}}\right\}$, we get that $M \cup J \cup M_{1}^{\prime} \cup \bar{M}_{2}^{\prime}$ is a model of

$$
\mathcal{M}[B]=K^{\prime} \wedge \bigwedge_{p \in V}\left(p_{e q} \rightarrow\left(p \equiv p^{\prime}\right)\right) \wedge \alpha,
$$


which in turn implies that $M \cup J$ is a model of $\exists V^{\prime} \mathcal{M}[B]$. On the other hand, since $E_{i_{0}}$ is a consistent belief change extension of $B$, Theorem 4.2 entails that $J$ is a model of $\mathcal{T}_{\text {ext }}[B]$. Consequently, $M \cup J$ is a model of $\mathcal{T}_{\text {ext }}[B] \wedge \exists V^{\prime} \mathcal{M}[B]$, and therefore

$$
\mathcal{T}_{m}[B]=\exists V_{e q}\left(\mathcal{T}_{\text {ext }}[B] \wedge \exists V^{\prime} \mathcal{M}[B]\right)
$$

is true under $M$.

Conversely, assume that $M \subseteq V$ is a model of $\mathcal{T}_{m}[B]$. Then, there is an interpretation $J \subseteq V_{e q}$ such that $M \cup J$ is a model of

$$
\mathcal{T}_{\text {ext }}[B] \wedge \exists V^{\prime} \mathcal{M}[B] .
$$

In particular, we have that $J$ is a model of $\mathcal{T}_{\text {ext }}[B]$, since the free variables of $\mathcal{T}_{\text {ext }}[B]$ are from $V_{e q}$. Hence, according to Theorem 4.2, we get that $E_{i_{0}}=C n\left(K^{\prime} \cup E Q_{i_{0}} \cup\{\alpha\}\right) \cap \mathcal{L}_{\mathcal{P}}$ is a consistent belief change extension of $B$, for $E Q_{i_{0}}=\left\{p \equiv p^{\prime} \mid p_{e q} \in J\right\}$. Since $K \dot{+} \alpha \subseteq E_{i_{0}}$, for showing that $M$ is a model of $K \dot{+} \alpha$, it suffi ces to show that $M$ is a model of $E_{l_{0}}$. This can be seen as follows.

Given that $M \cup J$ is a model of (13), we have that $M \cup J$ is a fortiori a model of $\exists V^{\prime} \mathcal{M}[B]$. Hence, there is some interpretation $N^{\prime} \subseteq V^{\prime}$ such that $M \cup J \cup N^{\prime}$ is a model of $\mathcal{M}[B]$. From this, we obtain that $M \cup N^{\prime}$ is a model of

$$
K^{\prime} \wedge \bigwedge_{\left(p \equiv p^{\prime}\right) \in E Q_{i_{0}}}\left(p \equiv p^{\prime}\right) \wedge \alpha,
$$

which in turn implies that $M \cup N^{\prime}$ is a model of $C n\left(K^{\prime} \cup E Q_{i_{0}} \cup\{\alpha\}\right)$. In particular, $M$ must be a model of all those elements from $C n\left(K^{\prime} \cup E Q_{i_{0}} \cup\{\alpha\}\right)$ which contain no atoms from $V^{\prime}$. In other words, $M$ is a model of $E_{i_{0}}=C n\left(K^{\prime} \cup E Q_{i_{0}} \cup\{\alpha\}\right) \cap \mathcal{L}_{\mathcal{P}}$.

2. Consider $B=(K, \emptyset,\{\neg \alpha\})$ in $\mathcal{L}_{\mathcal{P}}$, and assume that $M \subseteq V$ is a model of $K \dot{-} \alpha$. From Proposition 4.6, we obtain that there is some consistent belief change extension $E_{i_{0}}=C n\left(K^{\prime} \cup E Q_{i_{0}}\right) \cap \mathcal{L}_{\mathcal{P}}$ of $B$ and some $\pi_{i_{0}}^{k_{0}} \in \Pi_{i_{0}}$ such that $\lfloor K\rfloor_{i_{0}}^{k_{0}}$ is true under $M$, for $\Pi_{i_{0}}=\left\{\pi_{i_{0}}^{k} \mid \pi_{i_{0}}^{k}: \mathcal{P}_{\overline{E Q_{i}}} \rightarrow\{\top, \perp\}\right\}$. Analogous to Part 1, defi ne $M_{1}=M \cap \mathcal{P}_{E Q_{i_{0}}}$ and $M_{2}=M \backslash M_{1}$. Since $\lfloor K\rfloor_{i_{0}}^{k_{0}}$ is true under $M$, there is some $J \subseteq V \cap \mathcal{P}_{\overline{E Q}_{i_{0}}}$ such that $K$ is true under $M_{1} \cup J$, and therefore $M_{1}^{\prime} \cup J^{\prime}$ is a model of $K^{\prime}$. Hence, $M_{1} \cup M_{1}^{\prime} \cup J^{\prime}$ is a model of

$$
K^{\prime} \wedge \bigwedge_{p \equiv p^{\prime} \in E Q_{i_{0}}}\left(p \equiv p^{\prime}\right)
$$

Since no atoms from $V \cap \mathcal{P}_{\overline{E Q}_{i_{0}}}$ occur in (14), $M \cup M_{1}^{\prime} \cup J^{\prime}$ is also a model of (14). Applying similar arguments as in Part 1 , it follows that $M$ is a model of $\mathcal{T}_{m}[B]$.

The proof of the converse direction proceeds analogously to Part 1.

Proof 5.2 Since the membership relations are already dealt with in the main body of the paper, it remains to show that the problems EXT, CHOICE, and SKEPTICAL, as well as its variants for revision and contraction, are hard for the respective classes. 
1. REXT is NP-hard because a formula $\phi$ is satisfi able iff the belief change scenario $B_{R}=$ $(\{\phi\},\{\top\}, \emptyset)$ has a consistent belief change extension. Similarly, CEXT is NP-hard because $\phi$ is satisfi able iff $B_{C}=(\{\phi\}, \emptyset,\{\neg \perp\})$ possesses a consistent belief change extension. Either of these properties implies that EXT is NP-hard as well.

2. We show that RCHOICE and CCHOICE are $\Sigma_{2}^{P}$-hard; similar to the above, $\Sigma_{2}^{P}$-hardness of CHOICE is then an immediate consequence.

We fi rst deal with CCHOICE. According to Proposition 5.1, checking whether a closed QBF $\Phi$ of form $\exists P \forall Q \phi$, where $\phi$ is a propositional formula in disjunctive normal form and $P \cup$ $Q$ is a partition of $\operatorname{Var}(\phi)$, is $\Sigma_{2}^{P}$-hard. In order to show $\Sigma_{2}^{P}$-hardness of CCHOICE, we construct a polynomial-time transformation mapping each closed QBF $\Phi$ of the above form into a pair $\left(B_{C}, \phi^{*}\right)$, where $B_{C}=(K, \emptyset,\{\neg \alpha\})$ is a belief change scenario and $\phi^{*}$ is a formula, such that $\Phi$ is valid iff there is a consistent choice contraction $K{ }_{-}{ }_{c} \alpha$ containing $\phi^{*}$.

The construction of $K, \alpha$, and $\phi^{*}$ is as follows. For $P=\left\{p_{1}, \ldots, p_{n}\right\}$, let $R=\left\{r_{1}, \ldots, r_{n}\right\}$ be a set of new atoms not occurring in $\operatorname{Var}(\phi)$. Defi ne

$$
\begin{aligned}
K & =\left\{p_{i} \wedge r_{i} \mid i=1, \ldots, n\right\}, \quad \text { and } \\
\alpha & =\bigvee_{i=1}^{n}\left(p_{i} \wedge r_{i}\right),
\end{aligned}
$$

and let $\phi^{*}$ be the result of replacing in $\phi$ each literal $\neg p_{j}$, for $p_{j} \in P$, by $r_{j} \in R(1 \leq$ $j \leq n)$. We show that $\Phi=\exists P \forall Q \phi$ is valid iff there is a consistent belief change extension $C n\left(K^{\prime} \cup E Q_{i_{0}}\right) \cap \mathcal{L}_{\mathcal{P}}$ of $B_{C}=(K, \emptyset,\{\neg \alpha\})$ containing $\phi^{*}$.

To begin with, observe that, for any consistent belief change extension $C n\left(K^{\prime} \cup E Q\right) \cap \mathcal{L}_{\mathcal{P}}$ of $B_{C}$, it holds that

(*) either $p_{j} \equiv p_{j}^{\prime} \in E Q$ or $r_{j} \equiv r_{j}^{\prime} \in E Q$, but not both, for each $1 \leq j \leq n$.

Assume that $\exists P \forall Q \phi$ is valid. Then, there is an interpretation $S_{0} \subseteq P$ such that for each $U \subseteq Q, S_{0} \cup U$ is a model of $\phi$. By construction of $\phi^{*}$, it follows that $S_{0} \cup T_{0} \cup U$ is a model of $\phi^{*}$, for $T_{0}=\left\{r_{j} \in R \mid p_{j} \in P \backslash S_{0}\right\}$ and each $U \subseteq Q$. Now defi ne

$$
E Q_{i_{0}}=\left\{p_{j} \equiv p_{j}^{\prime} \mid p_{j} \in S_{0}\right\} \cup\left\{r_{j} \equiv r_{j}^{\prime} \mid p_{j} \in P \backslash S_{0}\right\}
$$

Clearly, $C n\left(K^{\prime} \cup E Q_{i_{0}}\right) \cap \mathcal{L}_{\mathcal{P}}$ is a consistent belief change extension of $B_{C}$. We claim that $\phi^{*} \in C n\left(K^{\prime} \cup E Q_{i_{0}}\right) \cap \mathcal{L}_{\mathcal{P}}$.

Since $\phi^{*} \in \mathcal{L}_{\mathcal{P}}$, it suffi ces to show that $K^{\prime} \cup E Q_{i_{0}} \vdash \phi^{*}$. Let $M^{\prime} \cup N$ be a model of $K^{\prime} \cup E Q_{i_{0}}$, where $M^{\prime} \subseteq P^{\prime} \cup R^{\prime}$ and $N \subseteq P \cup Q \cup R$. Defi ne $N_{1}=N \cap(P \cup R)$ and $N_{2}=N \cap Q$. Hence, $N=N_{1} \cup N_{2}$. Now, by defi nition of $K$ and $E Q_{i_{0}}$, it must hold that $N_{1}=S_{0} \cup T_{0}$. But $S_{0} \cup T_{0} \cup U$ is a model of $\phi^{*}$, for any $U \subseteq Q$; in particular, since $N_{2} \subseteq Q, S_{0} \cup T_{0} \cup N_{2}$ is a model of $\phi^{*}$. Therefore, $N=N_{1} \cup N_{2}$ is a model of $\phi^{*}$. Since $\phi^{*}$ contains no primed atoms, it follows that $M^{\prime} \cup N$ is also a model of $\phi^{*}$. This proves the relation $K^{\prime} \cup E Q_{i_{0}} \vdash \phi^{*}$. Hence, we showed that $\exists P \forall Q \phi$ is valid only if there is consistent belief change extension of $B_{C}$ containing $\phi^{*}$. 
Conversely, assume that $\phi^{*} \in C n\left(K^{\prime} \cup E Q_{i_{0}}\right) \cap \mathcal{L}_{\mathcal{P}}$ for some consistent belief change extension $C n\left(K^{\prime} \cup E Q_{i_{0}}\right) \cap \mathcal{L}_{\mathcal{P}}$ of $B_{C}=(K, \emptyset,\{\neg \alpha\})$. We show that $\exists P \forall Q \phi$ is valid.

Observe that, according to Condition (*), we have either $p_{j} \equiv p_{j}^{\prime} \in E Q_{i_{0}}$ or $r_{j} \equiv r_{j}^{\prime} \in E Q_{i_{0}}$, but not both. Defi ne

$$
\begin{aligned}
& S_{0}=\left\{p_{j} \in P \mid p_{j} \equiv p_{j}^{\prime} \in E Q_{i_{0}}\right\}, \quad \text { and } \\
& T_{0}=\left\{r_{j} \in R \mid r_{j} \equiv r_{j}^{\prime} \in E Q_{i_{0}}\right\},
\end{aligned}
$$

and let $W_{0}=S_{0} \cup T_{0}$. Clearly, $p_{j} \in S_{0}$ iff $r_{j} \notin T_{0}$, for each $1 \leq j \leq n$. Furthermore, $W_{0} \cup W_{0}^{\prime}$ is a model of $E Q_{i_{0}}$. But, since $\operatorname{Var}\left(E Q_{i_{0}}\right)=W_{0} \cup W_{0}^{\prime}$, and $W_{0}^{\prime} \subseteq P^{\prime} \cup R^{\prime}$, we have that $W_{0} \cup P^{\prime} \cup R^{\prime}$ is a model of $E Q_{i_{0}}$, as well. Moreover, $W_{0} \cup P^{\prime} \cup R^{\prime}$ is a model of $K^{\prime} \cup E Q_{i_{0}}$. In fact, for any $U \subseteq Q, W_{0} \cup P^{\prime} \cup R^{\prime} \cup U$ is a model of $K^{\prime} \cup E Q_{i_{0}}$. Since, by hypothesis, $K^{\prime} \cup E Q_{i_{0}} \vdash \phi^{*}$, it follows that $W_{0} \cup P^{\prime} \cup R^{\prime} \cup U$ is a model of $\phi^{*}$, for any $U \subseteq Q$. But $\phi^{*}$ does not contain any primed atoms, so $W_{0} \cup U=S_{0} \cup T_{0} \cup U$ must also be a model of $\phi^{*}$, for any $U \subseteq Q$. Hence, by defi nition of $\phi^{*}$, and since $p_{j} \in S_{0}$ iff $r_{j} \notin T_{0}$, we obtain that $S_{0} \cup U$ is a model $\phi$, for each $U \subseteq Q$. We just proved that there is some $S_{0} \subseteq P$ such that for each $U \subseteq Q, \phi$ is true under $S_{0} \cup U$. This means that $\exists P \forall Q \phi$ is valid.

Now we deal with the case of RCHOICE. Consider $\Phi, K, \alpha$, and $\phi^{*}$ as above. We claim that $\Phi=\exists P \forall Q \phi$ is valid iff there is a consistent choice revision $K \dot{+}_{c}(\neg \alpha)$ containing $\phi^{*}$. From this, $\Sigma_{2}^{P}$-hardness of RCHOICE is an immediate consequence.

To prove the claim, we must show that $\Phi$ is valid iff there is a consistent belief change extension $C n\left(K^{\prime} \cup E Q_{i_{0}} \cup\{\neg \alpha\}\right) \cap \mathcal{L}_{\mathcal{P}}$ of $B_{R}=(K,\{\neg \alpha\}, \emptyset)$ containing $\phi^{*}$. Recall that we demonstrated above that $\Phi$ is valid iff there is a consistent belief change extension $C n\left(K^{\prime} \cup E Q_{i_{0}}\right) \cap \mathcal{L}_{\mathcal{P}}$ of $B_{C}=(K, \emptyset,\{\neg \alpha\})$ containing $\phi^{*}$. Furthermore, for any $E Q \subseteq\left\{v \equiv v^{\prime} \mid v \in P \cup R\right\}$, it holds that $C n\left(K^{\prime} \cup E Q\right) \cap \mathcal{L}_{\mathcal{P}}$ is a consistent belief change extension of $B_{C}=(K, \emptyset,\{\neg \alpha\})$ iff $C n\left(K^{\prime} \cup E Q \cup\{\neg \alpha\}\right) \cap \mathcal{L}_{\mathcal{P}}$ is a consistent belief change extension of $B_{R}=(K,\{\neg \alpha\}, \emptyset)$ (cf. also Theorem 4.1 of [12]). Hence, it suffi ces to show that the following condition holds:

$(* *) \phi^{*} \in C n\left(K^{\prime} \cup E Q\right) \cap \mathcal{L}_{\mathcal{P}}$ iff $\phi^{*} \in C n\left(K^{\prime} \cup E Q \cup\{\neg \alpha\}\right) \cap \mathcal{L}_{\mathcal{P}}$, for any consistent belief change extension $C n\left(K^{\prime} \cup E Q\right) \cap \mathcal{L}_{\mathcal{P}}$ of $B_{C}$.

Consider some consistent belief change extension $C n\left(K^{\prime} \cup E Q\right) \cap \mathcal{L}_{\mathcal{P}}$ of $B_{C}$. If $\phi^{*} \in$ $C n\left(K^{\prime} \cup E Q\right) \cap \mathcal{L}_{\mathcal{P}}$, then $\phi^{*} \in C n\left(K^{\prime} \cup E Q \cup\{\neg \alpha\}\right) \cap \mathcal{L}_{\mathcal{P}}$, by monotonicity of $C n(\cdot)$. So suppose $\phi^{*} \in C n\left(K^{\prime} \cup E Q \cup\{\neg \alpha\}\right) \cap \mathcal{L}_{\mathcal{P}}$. We must show that $K^{\prime} \cup E Q \vdash \phi^{*}$ holds.

Let $M^{\prime} \cup N$ be some model of $K^{\prime} \cup E Q$, where $M^{\prime} \subseteq P^{\prime} \cup R^{\prime}$ and $N \subseteq P \cup R \cup Q$. Since $C n\left(K^{\prime} \cup E Q\right) \cap \mathcal{L}_{\mathcal{P}}$ is a consistent belief change extension of $B_{C}, E Q$ satisfi es Condition (*), i.e., for $P=\left\{p_{1}, \ldots, p_{n}\right\}$ and $R=\left\{r_{1}, \ldots, r_{n}\right\}$, we have that $p_{j} \equiv p_{j}^{\prime} \in E Q$ or $r_{j} \equiv r_{j}^{\prime} \in$ $E Q$, but not both, for each $1 \leq j \leq n$. Defi ne

$$
\hat{N}=N \backslash\left(\left\{p_{j} \mid r_{j} \equiv r_{j}^{\prime} \in E Q\right\} \cup\left\{r_{j} \mid p_{j} \equiv p_{j}^{\prime} \in E Q\right\}\right) .
$$

Obviously, $M^{\prime} \cup \hat{N}$ is a model of

$$
K^{\prime} \cup E Q \cup\{\neg \alpha\}=\left\{p_{i}^{\prime} \wedge r_{i}^{\prime} \mid i=1, \ldots, n\right\} \cup E Q \cup \bigwedge_{i=1}^{n}\left(\neg p_{i} \vee \neg r_{i}\right)
$$


Hence, since $\phi^{*} \in C n\left(K^{\prime} \cup E Q \cup\{\neg \alpha\}\right), M^{\prime} \cup \hat{N}$ is a model of $\phi^{*}$. Moreover, since $\phi^{*}$ is a formula in disjunctive normal form, and no atom from $P$ or $R$ occurs negated in $\phi^{*}$, it follows that, for any $S \subseteq P$ and any $T \subseteq R, M^{\prime} \cup \hat{N} \cup S \cup T$ is also a model of $\phi^{*}$. In particular, $M^{\prime} \cup N$ is a model of $\phi^{*}$. This proves $K^{\prime} \cup E Q \vdash \phi^{*}$.

3. Again, we only show $\Pi_{2}^{P}$-hardness of RSKeptiCAL and CSKEPTICAL. To this end, we exploit some results by Eiter and Gottlob [16] as well as by Delgrande and Schaub [11, 12].

Let $P=\left\{p_{1}, \ldots, p_{n}\right\}$ and $Q=\left\{q_{1}, \ldots, q_{m}\right\}$ be two distinct sets of variables, and consider a closed QBF $\Phi$ of form $\forall P \exists Q \phi$, where $\phi$ is a propositional formula such that $P \cup Q=$ $\operatorname{Var}(\phi)$. Furthermore, let $R=\left\{r_{1}, \ldots, r_{n}\right\}$ be a set of variables distinct from $\operatorname{Var}(\phi)$, and let $v$ be a further variable not occurring in $\operatorname{Var}(\phi)$ or $R$. For $K$ and $\alpha$ as defi ned in the proof of CCHOICE above, defi ne the following knowledge base $K_{S}$ and formulas $\beta$ and $\gamma$ :

$$
\begin{aligned}
K_{S} & =K \cup Q \cup\{v\}, \\
\beta & =\neg \alpha \wedge(v \rightarrow \phi) \wedge\left(\left(q_{1} \vee \ldots \vee q_{m}\right) \rightarrow v\right), \quad \text { and } \\
\gamma & =\bigwedge_{i=1}^{n}\left(p_{i} \vee r_{i}\right) .
\end{aligned}
$$

As shown by Eiter and Gottlob [16], $\Phi$ is valid iff $v \in K_{S} \dot{+}{ }_{s}(\beta \wedge \gamma)$, where $\dot{+}_{s}$ is the Satoh revision operator. Furthermore, Delgrande and Schaub [11, 12] showed that $K_{S} \dot{+}_{s}(\beta \wedge \gamma)$ is equivalent to $K_{S} \dot{+}(\beta \wedge \gamma)$. Hence, we get that

$$
\Phi \text { is valid iff } v \in K_{S} \dot{+}(\beta \wedge \gamma) \text {. }
$$

Consequently, RSKEPTICAL is $\Pi_{2}^{P}$-hard.

As for CSKEPTICAL, one can show that $v \in K_{S} \dot{\dot{ }}(\beta \wedge \gamma)$ iff $v \in K_{S} \dot{-}(\neg \beta \vee \neg \gamma)$. Hence, in view of (15), $\Pi_{2}^{P}$-hardness of CSKEPTICAL is an immediate consequence.

Proof 5.3 $\mathrm{D}^{P}$-membership can be seen as follows. According to Proposition 4.1, given a belief change scenario $B=(K, R, C)$ and some set $E Q \subseteq\left\{p \equiv p^{\prime} \mid p \in \operatorname{Var}(B)\right\}$ of equivalences, deciding whether $E Q$ determines a consistent belief change extension of $B$ is equivalent to

(i) deciding whether $K^{\prime} \cup E Q \cup R \cup C$ is consistent, and

(ii) deciding whether $K^{\prime} \cup E Q \cup\left(p \equiv p^{\prime}\right) \cup R \cup C$ is inconsistent, for each $p \in \operatorname{Var}(B)$ such that $\left(p \equiv p^{\prime}\right) \notin E Q$.

Clearly, Task (i) is in NP and Task (ii) is in co-NP. Hence, the combined problem is in $\mathrm{D}^{P}$. For showing $\mathrm{D}^{P}$-hardness, we consider the following well-known $\mathrm{D}^{P}$-complete problem [40]:

SAT-UNSAT: Given two propositional formulas $\phi$ and $\psi$, decide whether $\phi$ is satisfi able and $\psi$ is unsatisfi able. 
We construct a polynomial transformation mapping each pair $(\phi, \psi)$ of propositional formulas into a belief change scenario $B$ and some set $E Q \subseteq\left\{p \equiv p^{\prime} \mid p \in \operatorname{Var}(B)\right\}$ of equivalences such that

(*) if $\phi$ is satisfi able and $\psi$ is unsatisfi able, then $E Q$ determines a consistent belief change scenario of $B$, and vice versa.

The construction of $B$ and $E Q$ is as follows. Let $\phi$ and $\psi$ be propositional formulas. Without loss of generality, we can assume that $\operatorname{Var}(\phi)$ and $\operatorname{Var}(\psi)$ are disjoint. Furthermore, let $p$ and $q$ be distinct atoms not occurring in $\operatorname{Var}(\phi) \cup \operatorname{Var}(\psi)$. Then, defi ne

$$
\begin{aligned}
B & =(\{p \rightarrow \phi, q \rightarrow \psi\},\{p, q\}, \emptyset), \quad \text { and } \\
E Q & =\left\{p \equiv p^{\prime}\right\} \cup\left\{v \equiv v^{\prime} \mid v \in \operatorname{Var}(\phi) \cup \operatorname{Var}(\psi)\right\} .
\end{aligned}
$$

It is easy to see that this construction obeys Condition $(*)$.

\section{References}

[1] C. Alchourrón, P. Gärdenfors, and D. Makinson. On the logic of theory change: Partial meet contraction and revision functions. Journal of Symbolic Logic, 50:510-530, 1985.

[2] S. Benferhat, D. Dubois, and H. Prade. A computational model for belief change and fusing ordered belief bases. In M.-A. Williams and H. Rott, editors, Frontiers in Belief Revision, volume 22 of Applied Logic Series, pages 109-134. Kluwer Academic Publishers, 2001.

[3] A. Borgida. Language features for flexible handling of exceptions in information systems. ACM Transactions on Database Systems, 10(4):565-603, 1985.

[4] M. Cadoli, A. Giovanardi, and M. Schaerf. An algorithm to evaluate quantifi ed Boolean formulae. In Proceedings of the 15th National Conference on Artificial Intelligence (AAAI '98), pages 262-267, Madison, Wisconsin, 1998.

[5] T. Chou and M. Winslett. A model-based belief revision system. Journal of Automated Reasoning, 12(2):157-208, 1994.

[6] A. Church. Introduction to Mathematical Logic, Volume I. Princeton University Press, 1956.

[7] M. Dalal. Investigations into theory of knowledge base revision. In Proceedings of the Seventh National Conference on Artificial Intelligence (AAAI '88), pages 449-479, St. Paul, Minnesota, 1988.

[8] J. de Kleer. An assumption-based TMS. Artificial Intelligence, 28:127-162, 1986.

[9] A. del Val. Syntactic characterizations of belief change operators. In Proceedings of the 13th International Joint Conference on Artificial Intelligence (IJCAI '93), pages 540-545, Chambéry, France, 1993. 
[10] J. Delgrande, A. Hunter, and T. Schaub. COBA: A consistency-based belief revision system. In S. Greco and N. Leone, editors, Proceedings of the Eighth European Conference on Logics in Artificial Intelligence (JELIA 2002), volume 2424 of Lecture Notes in Artificial Intelligence, pages 509-512. Springer-Verlag, 2002.

[11] J. Delgrande and T. Schaub. A consistency-based model for belief change: Preliminary report. In Proceedings of the 17th National Conference on Artificial Intelligence (AAAI 2000), pages 392-398, Austin, Texas, 2000.

[12] J. Delgrande and T. Schaub. A consistency-based approach for belief change. Artificial Intelligence, 151(1-2):1-41, 2003.

[13] E. Eder. Relative Complexities of First-Order Calculi. Artifi cial Intelligence. Vieweg Verlag, 1992.

[14] U. Egly, T. Eiter, V. Klotz, H. Tompits, and S. Woltran. Computing stable models with quantifi ed Boolean formulas: Some experimental results. In Proceedings of the 2001 AAAI Spring Symposium on Answer Set Programming, pages 53-59, 2001.

[15] U. Egly, T. Eiter, H. Tompits, and S. Woltran. Solving advanced reasoning tasks using quantifi ed Boolean formulas. In Proceedings of the 17th National Conference on Artificial Intelligence (AAAI 2000), pages 417-422, Austin, Texas, 2000.

[16] T. Eiter and G. Gottlob. On the complexity of propositional knowledge base revision, updates, and counterfactuals. Artificial Intelligence, 57:227-270, 1992.

[17] T. Eiter, V. Klotz, H. Tompits, and S. Woltran. Modal nonmonotonic logics revisited: Effi cient encodings for the basic reasoning tasks. In Proceedings of the Eleventh Conference on Automated Reasoning with Analytic Tableaux and Related Methods (TABLEAUX 2002), volume 2381 of Lecture Notes in Computer Science, pages 100-114. Springer-Verlag, 2002.

[18] R. Fagin, J. Ullman, and M. Vardi. On the semantics of updates in databases. Preliminary report. In Proceedings of the Second ACM Symposium on Principles of Database Systems SIGACT-SIGMOD, pages 352-365, 1983.

[19] R. Feldmann, B. Monien, and S. Schamberger. A distributed algorithm to evaluate quantifi ed Boolean formulas. In Proceedings of the 17th National Conference on Artificial Intelligence (AAAI 2000), pages 285-290, Austin, Texas, 2000.

[20] K. Forbus. Introducing actions into qualitative simulation. In Proceedings of the Eleventh International Joint Conference on Artificial Intelligence (IJCAI '89), pages 1273-1278, Los Angeles, California, 1989.

[21] P. Gärdenfors. Knowledge in Flux: Modelling the Dynamics of Epistemic States. The MIT Press, Cambridge, MA, 1988.

[22] M. R. Garey and D. S. Johnson. Computers and Intractability. W. H. Freeman, 1979.

[23] M. Ginsberg. Counterfactuals. Artificial Intelligence, 30:35-79, 1986. 
[24] E. Giunchiglia, M. Narizzano, and A. Tacchella. QUBE: A system for deciding quantifi ed Boolean formulas satisfi ability. In Proceedings of the First International Joint Conference on Automated Reasoning (IJCAR 2001), volume 2083 of Lecture Notes in Artificial Intelligence, pages 364-369. Springer-Verlag, 2001.

[25] H. Katsuno and A. Mendelzon. On the difference between updating a knowledge base and revising it. In P. Gärdenfors, editor, Belief Revision, pages 183-203. Cambridge University Press, 1992.

[26] H. Kautz and B. Selman. Planning as satisfi ability. In Proceedings of the Tenth European Conference on Artificial Intelligence (ECAI '92), pages 359-363, Vienna, Austria, 1992.

[27] H. Kleine-Büning, M. Karpinski, and A. Flögel. Resolution for quantifi ed Boolean formulas. Information and Computation, 117(1):12-18, 1995.

[28] S. Konieczny and R. Pino Pérez. Merging information under constraints: A logical framework. Journal of Logic and Computation, 12(5):773-808, 2002.

[29] S. Leśniewski. Grundzüge eines neuen Systems der Grundlagen der Mathematik. Fundamenta Mathematica, 14:1-81, 1929.

[30] R. Letz. Lemma and model caching in decision procedures for quantifi ed Boolean formulas. In Proceedings of the Eleventh Conference on Automated Reasoning with Analytic Tableaux and Related Methods (TABLEAUX 2002), volume 2381 of Lecture Notes in Computer Science, pages 160-175. Springer-Verlag, 2002.

[31] P. Liberatore and M. Schaerf. Relating belief revision and circumscription. In Proceedings of the 14th International Joint Conference on Artificial Intelligence (IJCAI '95), pages 15571563, Montréal, Canada, 1995. Morgan Kaufmann.

[32] P. Liberatore and M. Schaerf. Reducing belief revision to circumscription (and vice versa). Artificial Intelligence, 93(1-2):261-296, 1997.

[33] P. Liberatore and M. Schaerf. Arbitration (or how to merge knowledge bases). IEEE Transactions on Knowledge and Data Engineering, 10(1):76-90, 1998.

[34] V. Lifschitz. Circumscription. In D. M. Gabbay, C. J. Hogger, and J. A. Robinson, editors, Handbook of Logic in Artificial Intelligence and Logic Programming, pages 297-352. Clarendon Press, 1994.

[35] J. Łukasiewicz and A. Tarski. Untersuchungen über den Aussagenkalkül. Comptes Rendus Séances Société des Sciences et Lettres Varsovie, 23(Cl. III):30-50, 1930.

[36] D. Makinson. How to give it up: A survey of some formal aspects of the logic of theory change. Synthese, 62:347-363, 1985.

[37] J. McCarthy. Circumscription - a form of nonmonotonic reasoning. Artificial Intelligence, 13:27-39, 1980. 
[38] A. R. Meyer and L. J. Stockmeyer. Word problems requiring exponential time. In ACM Symposium on Theory of Computing (STOC '73), pages 1-9. ACM Press, 1973.

[39] B. Nebel. Syntax based approaches to belief revision. In P. Gärdenfors, editor, Belief Revision, pages 52-88. Cambridge University Press, 1992.

[40] C. Papadimitriou. Computational Complexity. Addison-Wesley, Reading, Mass., 1994.

[41] D. Pearce, H. Tompits, and S. Woltran. Encodings for equilibrium logic and logic programs with nested expressions. In Proceedings of the Tenth Portuguese Conference on Artificial Intelligence (EPIA 2001), volume 2258 of Lecture Notes in Artificial Intelligence, pages 306320. Springer-Verlag, 2001.

[42] D. A. Plaisted and S. Greenbaum. A structure preserving clause form translation. Journal of Symbolic Computation, 2(3):293-304, 1986.

[43] D. Poole. A logical framework for default reasoning. Artificial Intelligence, 36(1):270-48, 1988.

[44] R. Reiter. A theory of diagnosis from fi rst principles. Artificial Intelligence, 32(1):57-96, 1987.

[45] J. Rintanen. Constructing conditional plans by a theorem prover. Journal of Artificial Intelligence Research, 10:323-352, 1999.

[46] J. Rintanen. Improvements to the evaluation of quantifi ed Boolean formulae. In Proceedings of the 16th International Joint Conference on Artificial Intelligence (IJCAI '99), pages 11921197, Stockholm, Sweden, 1999.

[47] B. Russell. The theory of implication. American Journal of Mathematics, 28(2):159-202, 1906.

[48] K. Satoh. Nonmonotonic reasoning by minimal belief revision. In Proceedings of the International Conference on Fifth Generation Computer Systems, pages 455-462, Tokyo, 1988.

[49] J. Srzednicki and Z. Stachniak, editors. Lesniewski’s Systems Protothetic. Dordrecht, 1998.

[50] L. J. Stockmeyer. The polynomial-time hierarchy. Theoretical Computer Science, 3(1):1-22, 1976.

[51] H. Tompits. Expressing default abduction problems as quantifi ed Boolean formulas. AI Communications, 16:89-105, 2003.

[52] R. Wassermann. Resource-Bounded Belief Revision. PhD thesis, Institute for Logic, Language, and Computation, University of Amsterdam, 1999.

[53] A. Weber. Updating propositional formulas. In Proceedings of the First Conference on Expert Database Systems, pages 487-500, 1986. 
[54] A. N. Whitehead and B. Russell. Principia Mathematica, volume 1-3. Cambridge University Press, 1910-13.

[55] M.-A. Williams. Theory base change: A computational model. In Proceedings of the 14th International Joint Conference on Artificial Intelligence (IJCAI '95), pages 1541-1547, Montréal, Canada, 1995.

[56] M. Winslett. Reasoning about action using a possible models approach. In Proceedings of the Seventh National Conference on Artificial Intelligence (AAAI'88), pages 89-93, St. Paul, Minnesota, 1988.

[57] M. Winslett. Sometimes updates are circumscription. In N. S. Sridharan, editor, Proceedings of the Eleventh International Joint Conference on Artificial Intelligence (IJCAI '89), pages 859-863, Detroit, Michigan, 1989. Morgan Kaufmann.

[58] M. Winslett. Updating Logical Databases. Cambridge University Press, 1990.

[59] C. Wrathall. Complete sets and the polynomial-time hierarchy. Theoretical Computer Science, 3(1):23-33, 1976. 